\title{
Statistical characteristics of AGW wave packet propagation in the lower atmosphere observed by the MU radar
}

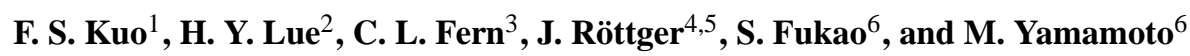 \\ ${ }^{1}$ Department of Electro-Optical Engineering, Vanung University, Chung-Li, Taiwan \\ ${ }^{2}$ Department of Physics, Fu Jen University, Hsin Chuang, Taiwan \\ ${ }^{3}$ Graduate School of Materials, National Yunlin University of Science and Technology, Douliou, Yunlin, Taiwan \\ ${ }^{4}$ MPI for Solar System Research, Katlenburg-Lindau, Germany \\ ${ }^{5}$ Institute of Space Science, National Central University, Chung-Li, Taiwan \\ ${ }^{6}$ Research Institute for Sustainable Humanosphere, Kyoto University, Kyoto, Japan
}

Received: 15 March 2009 - Revised: 5 August 2009 - Accepted: 3 September 2009 - Published: 2 October 2009

\begin{abstract}
We study the horizontal structure of the atmospheric gravity waves (AGW) in the height ranges between 6 and $22 \mathrm{~km}$ observed using the MU radar at Shigaraki in Japan, during a 3 day period in January and a 4 day period in August 1988. The data were divided by double Fourier transformation into a data set of upward moving waves and a data set of downward moving waves for independent analysis. The phase and group velocity tracing technique was applied to measure the vertical group and phase velocity as well as the characteristic period of the gravity wave packet. Then the dispersion equation of the linear theory of AGW was solved to obtain its intrinsic wave period - horizontal wavelength and horizontal group velocity - and the vertical flux of horizontal momentum associated with each wave packet was estimated to help determine the direction of the characteristic horizontal wave vector. The results showed that the waves with periods in the range of $30 \mathrm{~min} \sim 6 \mathrm{~h}$ had horizontal scales ranging from $20 \mathrm{~km}$ to $1500 \mathrm{~km}$, vertical scales from $4 \mathrm{~km}$ to $15 \mathrm{~km}$, and horizontal phase velocities from $15 \mathrm{~m} / \mathrm{s}$ to $60 \mathrm{~m} / \mathrm{s}$. The upward moving wave packets of wave period of $2 \mathrm{~h} \sim 6 \mathrm{~h}$ had horizontal group velocities mainly toward eastsouth-east and northeast in winter, and mainly in the section between the directions of west-north-west and north in summer.
\end{abstract}

Keywords. Meteorology and atmospheric dynamics (Middle atmosphere dynamics; Waves and tides; Instruments and techniques)

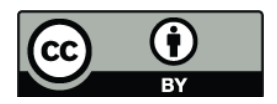

Correspondence to: F. S. Kuo (fskuo@mail.vnu.edu.tw)

\section{Introduction}

The behaviour of a gravity wave is widely recognized in playing an important role in transporting energy and momentum in the middle atmosphere. One of the important characteristics of gravity waves is the horizontal structure. A number of groups have tried to detect horizontal phase velocities and propagation directions of gravity waves with ground-based radar at a single location. Vincent and Reid (1983) determined typical values of zonal wavelength and zonal phase velocity at $80-94 \mathrm{~km}$ altitude to be about $70 \mathrm{~km}$ and $70 \mathrm{~m} / \mathrm{s}$, respectively, utilizing multiple Doppler beams observations with the Adelaide MF radar. Meek et al. (1985) and Manson and Meek (1988) observed gravity waves at 60$110 \mathrm{~km}$ altitude to find that the gravity waves with intrinsic periods of 10 to $200 \mathrm{~min}$ had horizontal scales ranging from 44 to $1000 \mathrm{~km}$ and phase velocities from 20 to $93 \mathrm{~m} / \mathrm{s}$. From meteor radar observations at $90-100 \mathrm{~km}$ altitude, Yamamoto et al. (1986) determined that the gravity waves with periods of 2 to $8 \mathrm{~h}$ had mean zonal phase velocity of $\sim 25 \mathrm{~m} / \mathrm{s}$ and a wavelength of $\sim 360 \mathrm{~km}$; and further they found that the westward propagating waves were dominant in winter. Nakamura et al. (1993) observed gravity waves in the $70-75 \mathrm{~km}$ altitude range by MU radar, and found that the mean values of the vertical wavelength, horizontal wavelength and intrinsic period of the gravity waves were $8.2 \mathrm{~km}, 1200 \mathrm{~km}$, and $10.2 \mathrm{~h}$, respectively, the mean horizontal phase velocity was $33 \mathrm{~m} / \mathrm{s}$, and the horizontal propagation direction was generally eastward throughout the year except for the westward propagation in early winter (November to December). Ebel et al. (1987) found that the wind perturbation in the height range of 61 to $105 \mathrm{~km}$ was polarized toward the north-south

Published by Copernicus Publications on behalf of the European Geosciences Union. 
direction with significant seasonal variation. Manson and Meek (1988) found that horizontal propagation of gravity waves in the height range of $64 \sim 97 \mathrm{~km}$ was mainly toward the south, while eastward and westward momentum fluxes were observed in summer and winter, respectively. Radar observations by Vincent and Fritts (1987) and rocket observations by Eckermann and Vincent (1989) in the Southern Hemisphere revealed some difference in the distribution of propagation directions between summer and winter. Tsuda et al. (1990) found that the preferential direction of the dominant gravity wave component at $60-90 \mathrm{~km}$ altitude in October 1986 over Shigaraki Japan was south-southeastward. Gavrilov et al. (1997) analyzed the internal gravity waves (IGWs) in the altitude range of $65-85 \mathrm{~km}$ over Shigaraki Japan by statistical methods; and decided that the propagation direction was mainly eastward in summer and westward in winter, they also decided that most of IGWs have momentum fluxes directed to the northeast in winter and to the east in summer.

In the lower stratosphere, a lot of IGWs propagating meridionally were reported by several case studies (e.g. Hirota and Niki, 1986; Yamanaka et al., 1989). Analyzing the dominant component of IGWs with short vertical wave length $(\leq 4 \mathrm{~km})$ and long ground-based period $(\geq 10 \mathrm{~h})$ in the lower stratosphere and upper troposphere over Shigaraki Japan, Sato (1994) found that in winter IGWs propagated generally southward in the $12-18 \mathrm{~km}$ region, while westward IGWs were dominant at $18-22 \mathrm{~km}$. In contrast to these observations (Hirota and Niki, 1986; Yamanaka et al., 1989; Sato, 1994) of IGWs propagating transverse to the jet stream, radar measurements (Thomas et al., 1999) at Aberystwyth $\left(52.4^{\circ} \mathrm{N}, 4.1^{\circ} \mathrm{W}\right)$ of winds at tropospheric and lower stratospheric heights indicated inertia-gravity waves propagating in a direction similar to that of the jet stream but at smaller velocities.

All these previous works seemed to regard the direction of AGW horizontal phase velocity as its horizontal propagation direction. But the wave transports its energy and momentum at its group velocity (not phase velocity), and the direction of the horizontal group velocity of the gravity wave is generally different from the direction of its horizontal wave vector when the background wind can not be ignored (see Appendix B). Therefore, analysis of the group velocities is necessary to understand the characteristics of gravity wave propagation. A method of wave packet analysis called phase- and group-velocity-tracing (Kuo et al., 1998) was developed to measure the group velocity, phase velocity along any specific direction and the characteristic wave period of a wave packet of any kind of wave. And this method was further proved by numerical simulation to be reasonably accurate in measuring the phase- and group- velocities of the projected wave packet motion of gravity wave along any oblique radar beam (Kuo et al., 2007). Once the vertical group and phase velocities $\left(v_{g z}\right.$ and $\left.v_{p z}\right)$ as well as the wave period $\tau$ of a gravity wave packet are determined, the horizontal group velocity and wavelength can be obtained by the dispersion equation. In this paper, we shall extend this technique to study the horizontal propagation of gravity waves in the lower atmosphere.

\section{Data and analysis procedure}

\subsection{Data}

Two data sets were analyzed in this study. One data set (called winter data set) of the wind velocity was taken during a three day period in January 1988 by the MU radar $\left(35^{\circ} \mathrm{N}\right.$, $136^{\circ} \mathrm{E}$ ) at Shigaraki, Japan; another data set (called summer data set) was taken by the same radar in the same year during a four day period in August. In every inter-pulse period, the radar antenna beam was steered sequentially toward the vertical and oblique directions at a zenith angle of $10^{\circ}$ (vertical $\rightarrow$ north $\rightarrow$ east $\rightarrow$ south $\rightarrow$ west). The beam width was 3 degrees, and the aspect sensitivity was negligible at the 10 degree zenith angle. The other observation parameters were: observation range $z=5 \sim 24 \mathrm{~km}$, range resolution $\Delta z=150 \mathrm{~m}$, time resolution $\Delta t=150 \mathrm{~s}$. There were some missing data points due to insufficient signal power or time breaks during the experiment operation. Before doing data analysis, the missing data was filled by interpolation from its neighbouring good data. By dual beam method, the zonal-velocity $u$, meridional velocity $v$, and vertical velocity $w$ were obtained from the Doppler velocities measured by the four oblique beams as described in our previous paper (Kuo et al., 2008). Figure 1 shows the $72 \mathrm{~h}$ averaged wind velocity profiles of winter data set and the $96 \mathrm{~h}$ averaged wind velocity profiles of summer data set. Winter data shows that a strong zonal wind jet existed in the height range $10-12 \mathrm{~km}$ with large vertical shear both above and below this jet. The meridional wind in winter was evidently much weaker than the zonal wind. No wind jet was found in the summer data. The zonal wind of the summer data was much weaker than that in the winter data, while the strength of the meridional wind of the summer data was comparable with that of the winter data. The frequency and vertical wave number spectra of this winter data were studied and presented in an earlier paper (Kuo et al., 1992); while the study of its vertical flux of horizontal momentum was presented in a recent paper (Kuo et al., 2008).

\subsection{Propagation parameters of wave packets calculated by dispersion equation}

The data was separated by double-Fourier transformation into a data set of waves with downward-phase-velocity and a data set of waves with upward-phase-velocity for wave packet analysis. Then the vertical group velocity $v_{g z}$, vertical phase velocity $v_{p z}$, and the characteristic wave period $\tau$ of a wave packet were measured directly by the technique of phase and group velocity tracing (Kuo et al., 1998, 2003, 
2007, 2008), and the observed wave frequency $\sigma$ and vertical wave number $m$ were obtained readily by $\sigma=1 / \tau$ and $m=\sigma / v_{p z}$, respectively. For the readers' convenience, we put the method of up-down wave separation in Sect. A1 and the technique of phase and group velocity tracing in Sect. A2 of Appendix A.

The AGW is characterized by the following dispersion equations (Fritts and Alexander, 2003),

$m^{2}=\frac{\left(k^{2}+\ell^{2}\right)\left(N^{2}-\omega^{2}\right)}{\left(\omega^{2}-f^{2}\right)}-\frac{1}{4 H^{2}}$,

$\omega=\sigma-\boldsymbol{u}_{h} \cdot \boldsymbol{k}_{h}=\sigma-k \bar{u}-\ell \bar{v}$

where $\boldsymbol{k}_{h}$ is the horizontal wave vector; $k, \ell, m$, are the zonal-, meridional-, and vertical- wave number; $\sigma, N, f$, and $\omega$ are the observed-, BV-, inertial- and intrinsic frequency respectively; $H$ is the scale length of the atmospheric density. The intrinsic frequency $\omega$ is the frequency that would be observed in a frame of reference moving with the background wind velocity $\boldsymbol{u}_{h}=(\bar{u}, \bar{v})$. The group velocity $\left(v_{g x}, v_{g y}, v_{g z}\right)$ of an AGW packet is given by (Fritts and Alexander, 2003),

$v_{g z}=\frac{-m\left(\omega^{2}-f^{2}\right)}{\omega\left(k^{2}+\ell^{2}+m^{2}+\frac{1}{4 H^{2}}\right)}$,

$v_{g x}=\bar{u}+\frac{k\left(N^{2}-\omega^{2}\right)}{\omega\left(k^{2}+\ell^{2}+m^{2}+\frac{1}{4 H^{2}}\right)}$,

$v_{g y}=\bar{v}+\frac{\ell\left(N^{2}-\omega^{2}\right)}{\omega\left(k^{2}+\ell^{2}+m^{2}+\frac{1}{4 H^{2}}\right)}$,

and the phase velocity $\left(v_{p x}, v_{p y}, v_{p z}\right)$ is defined by,

$$
\left(v_{p x}, v_{p y}, v_{p z}\right)=\left(\frac{\sigma}{k}, \frac{\sigma}{\ell}, \frac{\sigma}{m}\right) \text {. }
$$

Our sign convention here assumes that $\sigma$ is positively defined. When the characteristic intrinsic frequency $\omega$ is positive, the vertical group velocity $v_{g z}$ is downward for positive $m$ (upward phase velocity) and upward for negative $m$ (downward phase velocity); when $\omega$ is negative, $v_{g z}$ is upward for positive $m$ (upward phase velocity) and downward for negative $m$ (downward phase velocity). We call a wave packet with positive $\omega$ a type 1 wave packet, whose phase and energy have opposite sense of vertical propagation, and call a wave packet with negative $\omega$ a type 2 wave packet, whose phase and energy have the same sense of vertical propagation.

Given the atmospheric parameters $f, N, H$, and the background wind velocity $(\bar{u}, \bar{v})$, we may search the most probable characteristic horizontal wave vector $(k, \ell)$ of a wave packet to match its measured quantities $\sigma, m$, and $v_{g z}$ by the Eqs. (1 4) through following procedure: By assigning the horizontal phase speed $v_{p h}=\tilde{v}_{p h}$ and azimuth angle $\phi=\tilde{\phi}$

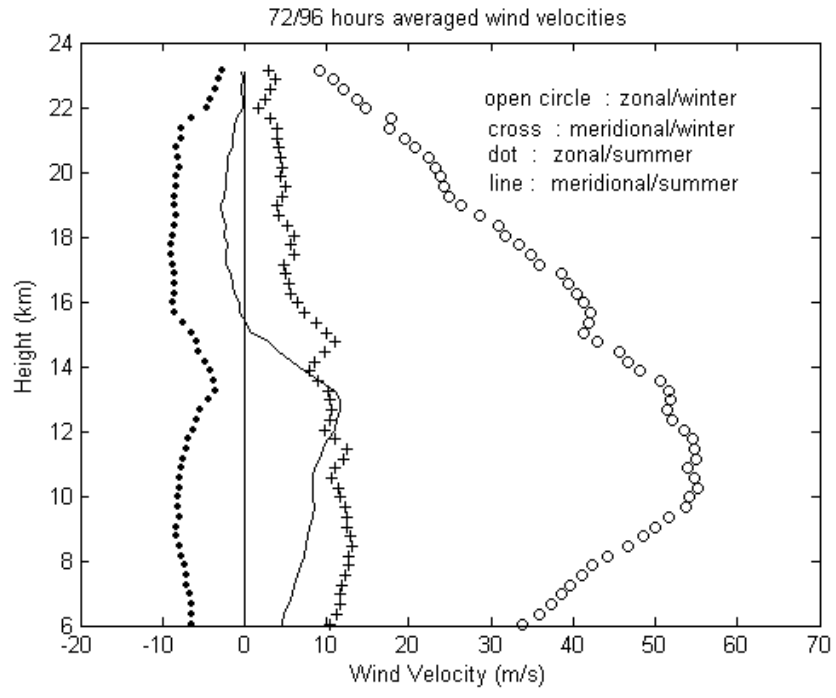

Fig. 1. Open circle: $72 \mathrm{~h}$ averaged velocity of winter zonal wind; Cross: $72 \mathrm{~h}$ averaged velocity of winter meridional wind; Dot: $96 \mathrm{~h}$ averaged velocity of summer zonal wind; Line: $96 \mathrm{~h}$ averaged velocity of summer meridional wind. The vertical straight line is the reference line for zero velocity.

to the wave packet, we obtain the corresponding horizontal wave vector $(\tilde{k}, \tilde{\ell})$ by Eq. (5),

$$
(\tilde{k}, \tilde{\ell})=\left(\sigma \cdot \sin \tilde{\phi} / \tilde{v}_{p h}, \sigma \cdot \cos \tilde{\phi} / \tilde{v}_{p h}\right) \text {. }
$$

Then, substitute $(\tilde{k}, \tilde{\ell})$ into Eqs. (1), (2), (3a) and (4) to obtain $\tilde{m}, \tilde{\omega}, \tilde{v}_{g z}$ and $\tilde{v}_{p z}$ respectively. The wave vector $(\tilde{k}, \tilde{\ell})$ is accepted as a possible characteristic wave vector of the wave packet if following conditions of Eq. (6) are satisfied,

$\left|\left(v_{g z}-\tilde{v}_{g z}\right) / v_{g z}\right|<0.1$ and $\left|\left(v_{p z}-\tilde{v}_{p z}\right) / v_{p z}\right|<0.05$

Otherwise, $(\tilde{k}, \tilde{\ell})$ is rejected. In this study, the following atmospheric parameters were used in the calculations: $\quad N=1.047 \times 10^{-2} \mathrm{~s}^{-1} \quad(10 \mathrm{~min} \quad$ BV-period $)$, $f=8.31 \times 10^{-5} \mathrm{~s}^{-1}(21 \mathrm{~h}$ inertial period $)$ and $H=8 \mathrm{~km}$; $(\bar{u}, \bar{v})=(37.8,8.1) \mathrm{m} / \mathrm{s}$ for the winter data, and $(\bar{u}, \bar{v})=(-8.46,3.08) \mathrm{m} / \mathrm{s}$ for the summer data (the method to decide these constant wind velocities will be explained in Sect. 3.1). Any combination pair of $\left(\tilde{v}_{p h}, \tilde{\phi}\right)$ from the following values was used to calculate the characteristic gravity wave parameters for each measured wave packet:

$\tilde{v}_{p h}=1,2,3,-------, 98,99,100 \mathrm{~m} / \mathrm{s}$

$\tilde{\phi}=0.5^{\circ}, 1.5^{\circ}, 2.5^{\circ},------, 357.5^{\circ}, 358.5^{\circ}, 359.5^{\circ}$.

Among all the possible characteristic wave vectors $(\tilde{k}, \tilde{\ell})$ (i.e., satisfying conditions 6 ), the one yielding the smallest error is regarded as the characteristic wave vector, and 
Table 1. Wave period bands and wave length bands corresponding to the frequency- and wave number- windows used to sample the wave packets for phase and group velocity tracing analysis.

\begin{tabular}{ccc}
\hline Windows & Wave period band & Wave length band \\
\hline T2Z3 & 30 min-2 h & $2.95-17.7 \mathrm{~km}$ \\
T3aZ3 & $2-7 \mathrm{~h}$ & $2.95-17.7 \mathrm{~km}$ \\
$\mathrm{~T} 3 \mathrm{bZ3}$ & $3-24 \mathrm{~h}$ & $2.95-17.7 \mathrm{~km}$ \\
$\mathrm{~T} 3 \mathrm{cZ3}$ & $4-24 \mathrm{~h}$ & $2.95-17.7 \mathrm{~km}$ \\
$\mathrm{~T} 4 Z 3$ & $7-24 \mathrm{~h}$ & $2.95-17.7 \mathrm{~km}$ \\
$\mathrm{~T} 2 Z 4$ & $30 \mathrm{~min}-2 \mathrm{~h}$ & $5.9-17.7 \mathrm{~km}$ \\
$\mathrm{~T} 3 \mathrm{aZ4}$ & $2-7 \mathrm{~h}$ & $5.9-17.7 \mathrm{~km}$ \\
$\mathrm{~T} 3 \mathrm{bZ4}$ & $3-24 \mathrm{~h}$ & $5.9-17.7 \mathrm{~km}$ \\
$\mathrm{~T} 3 \mathrm{cZ4}$ & $4-24 \mathrm{~h}$ & $5.9-17.7 \mathrm{~km}$ \\
$\mathrm{~T} 4 Z 4$ & $7-24 \mathrm{~h}$ & $5.9-17.7 \mathrm{~km}$ \\
$\mathrm{~T} 2 Z 5$ & $30 \mathrm{~min}-2 \mathrm{~h}$ & $8.85-17.7 \mathrm{~km}$ \\
$\mathrm{~T} 3 \mathrm{aZ5}$ & $2-7 \mathrm{~h}$ & $8.85-17.7 \mathrm{~km}$ \\
$\mathrm{~T} 3 \mathrm{bZ5}$ & $3-24 \mathrm{~h}$ & $8.85-17.7 \mathrm{~km}$ \\
$\mathrm{~T} 3 \mathrm{cZ5}$ & $4-24 \mathrm{~h}$ & $8.85-17.7 \mathrm{~km}$ \\
$\mathrm{~T} 4 Z 5$ & $7-24 \mathrm{~h}$ & $8.85-17.7 \mathrm{~km}$ \\
\hline
\end{tabular}

the corresponding wave packet is considered a "gravity wave packet". In case no value of $(\tilde{k}, \tilde{\ell})$ was found to satisfy condition (6), the corresponding wave packet will be rejected as a gravity wave packet. It is important to note that the second term in the right hand side of Eq. (2) is characterized by $\boldsymbol{k}_{h} \cdot \boldsymbol{u}_{h}=k_{h} u_{h} \cos \left(\phi_{k h}-\phi_{u h}\right)=k_{h} u_{h} \cos \left(\phi_{u h}-\phi_{k h}\right)$, where $k_{h}$ and $\phi_{k h}$ are the amplitude and the azimuth angle of the wave vector $\boldsymbol{k}_{h}$, respectively. If a horizontal wave vector with an amplitude of $k_{h}$ and azimuth angle of $\phi_{k h}=\phi_{u h}+\Delta \phi$ is a solution of the dispersion equation, then another horizontal wave vector with the same wave amplitude $k_{h}$ but different azimuth angle $\phi_{u h}-\Delta \phi$ is also a solution. These two solution-wave vectors are symmetric with respect to the mean wind velocity vector $\boldsymbol{u}_{h}$. Eventually we must decide which one of the two solution-wave vectors is more likely to be the true solution.

We will discuss the statistics of 1780 wave packets for summer data and 740 wave packets for winter data. These summer wave packets were obtained from both the zonal and meridional perturbation velocities, while the winter wave packets were obtained only from the zonal perturbation velocity because the zonal profile yields a stronger wave packet signal than the meridional profile. Also, only the upward packets of the winter data were included because the statistics of upward events and downward events were similar. However, both upward events and downward events of the summer data will be added together to yield better statistics. It is found that 838 out of 1780 upward and downward wave packets $(47.1 \%)$ of the summer data were identified as gravity wave packets; likewise, 586 out of 740 upward wave packets $(79.2 \%)$ of the winter data were identi-
Table 2. Number (in bold) of upward moving wave packets in each window identified to be atmospheric gravity waves by dispersion equation. The number under the slash represents the number of wave packets being measured in each respective window, and the percentage of the originally measured wave packets to be identified as gravity wave packets was listed under the respective numbers. The events were obtained from winter data set. The content of window T3bZ4 was displayed in the box of 3rd row-4th column.

\begin{tabular}{cccccc}
\hline Window & T2- & T3a- & T3b- & T3c- & T4 \\
\hline -Z3- & $\mathbf{1 4 8 / 2 0 6}$ & $\mathbf{5 7 / 7 9}$ & $\mathbf{1 6 / 1 9}$ & $\mathbf{1 6 / 1 8}$ & $\mathbf{7 / 1 8}$ \\
& $71.8 \%$ & $72.2 \%$ & $84.2 \%$ & $88.9 \%$ & $38.9 \%$ \\
$-Z 4-$ & $\mathbf{1 1 3 / 1 3 2}$ & $\mathbf{3 7 / 4 5}$ & $\mathbf{1 5 / 1 7}$ & $\mathbf{1 2} / 15$ & $\mathbf{1 3 / 1 4}$ \\
& $85.6 \%$ & $82.2 \%$ & $88.2 \%$ & $80.0 \%$ & $92.9 \%$ \\
- Z5 & $\mathbf{1 0 4 / 1 1 4}$ & $\mathbf{2 9 / 3 8}$ & $\mathbf{8} / 11$ & $\mathbf{4 / 4}$ & $\mathbf{7 / 1 0}$ \\
& $91.2 \%$ & $76.3 \%$ & $72.7 \%$ & $100 \%$ & $70 \%$ \\
\hline
\end{tabular}

fied as gravity wave packets. These wave packets were obtained from different windows as defined in Table 1, where the nomenclature of the windows is as follows: The letter $T(Z)$ stands for time (height), and the number after $T(Z)$ stands for different time range (height range). So from Table 1 , we can readily identify $\mathrm{T} 3 \mathrm{c}$ for example, to represent the time range of $4 \sim 24 \mathrm{~h}$ and $\mathrm{Z} 4$ to represent the height range of $5.9 \sim 17.7 \mathrm{~km}$. Different windows in Table 1 have different upper frequency and wave number boundaries, but may have the same lower frequency and wave number boundaries, because only the high frequency end (high wave number end) dictates the characteristic wave period (wavelength) of the wave packets as we had frequently explained in our previous papers (Kuo et al., 2003, 2008; Kuo and Röttger, 2005). The number of gravity wave packets (in bold) along with the original number of wave packets being measured (the number under the slash) in different window were listed in Table 2 (for winter data set) and Table 3 (for summer data set), where the content in the 5th column of the 4th row represents the content of window T3cZ5, and the 2 nd column of the 3rd row represents the content of window T2Z4, and so on. The percentage of the originally measured wave packets to be identified as gravity wave packets in each window was listed under the respective numbers. We noticed two points: 1, The winter data has a much higher percentage $(79.2 \%)$ of gravity wave packets than the summer data $(47.1 \%) ; 2$, For any fixed wave period, larger vertical wavelength waves have a higher percentage of gravity wave packets in general. The first point is probably because there was a much stronger wind jet in the winter than in the summer to generate wave activity. The second point might be due to the measurement error, because larger vertical wavelength waves have larger vertical phase- and group- velocity, thus will yield a smaller percentage error in measurement according to condition (6). 
Table 3. Sum of the numbers (in bold) of upward-moving and downward moving wave packets in each window identified to be atmospheric gravity waves by dispersion equation. The number under the slash represents the number of wave packets being measured in each respective window, and the percentage of the originally measured wave packets to be identified as gravity wave packets was listed under the respective numbers. The events were obtained from summer data set.

\begin{tabular}{cccccc}
\hline Window & T2- & T3a- & T3b- & T3c- & T4- \\
\hline -Z3- & $\mathbf{1 8 8} / 434$ & $\mathbf{4 2 / 1 2 5}$ & $\mathbf{3 7 / 1 0 8}$ & $\mathbf{1 8} / 72$ & $\mathbf{1 6 / 3 2}$ \\
& $43.3 \%$ & $33.6 \%$ & $34.3 \%$ & $25.0 \%$ & $50.0 \%$ \\
- Z4- & $\mathbf{1 5 2 / 3 1 3}$ & $\mathbf{3 9 / 9 7}$ & $\mathbf{3 5} / 81$ & $\mathbf{2 1 / 5 1}$ & $\mathbf{2 2 / 3 4}$ \\
& $48.6 \%$ & $40.2 \%$ & $43.2 \%$ & $41.2 \%$ & $64.7 \%$ \\
- Z5 & $\mathbf{1 4 4 / 2 1 1}$ & $\mathbf{4 3 / 9 5}$ & $\mathbf{3 4 / 6 3}$ & $\mathbf{2 3 / 3 7}$ & $\mathbf{2 4 / 2 7}$ \\
& $68.2 \%$ & $45.3 \%$ & $54.0 \%$ & $62.2 \%$ & $88.9 \%$ \\
\hline
\end{tabular}

Table 4. Mean \pm standard deviation of characteristic wave period $\tau$, vertical wave length $\lambda_{z}$, and horizontal wavelength $\lambda_{h}$ of the atmospheric gravity wave packets with upward group velocity. The events were from winter data set.

\begin{tabular}{cccc}
\hline Window & $\tau(\min )$ & $\lambda_{z}(\mathrm{~km})$ & $\lambda_{h}(\mathrm{~km})$ \\
\hline T2Z3 & $41.5 \pm 4.07$ & $4.99 \pm 1.04$ & $53.9 \pm 28.5$ \\
T3aZ3 & $145.9 \pm 12.8$ & $5.36 \pm 1.08$ & $264.9 \pm 103.1$ \\
T3bZ3 & $217.0 \pm 12.5$ & $5.29 \pm 1.14$ & $305.4 \pm 165.8$ \\
T3cZ3 & $286.5 \pm 27.7$ & $5.37 \pm 0.69$ & $329.5 \pm 132.3$ \\
T4Z3 & $524.6 \pm 61.4$ & $5.44 \pm 0.796$ & $611.4 \pm 184.5$ \\
T2Z4 & $42.2 \pm 5.29$ & $8.50 \pm 1.51$ & $62.7 \pm 30.7$ \\
T3aZ4 & $145.8 \pm 11.3$ & $8.29 \pm 1.00$ & $288.5 \pm 109.2$ \\
T3bZ4 & $222.4 \pm 23.4$ & $9.06 \pm 1.72$ & $398.6 \pm 156.0$ \\
T3cZ4 & $298.3 \pm 33.8$ & $8.17 \pm 1.19$ & $529.8 \pm 252.3$ \\
T4Z4 & $486.1 \pm 18.3$ & $9.22 \pm 1.59$ & $1301 \pm 425.8$ \\
T2Z5 & $42.6 \pm 5.28$ & $11.3 \pm 1.05$ & $63.3 \pm 33.9$ \\
T3aZ5 & $145.8 \pm 14.0$ & $12.0 \pm 1.19$ & $304.1 \pm 100.0$ \\
T3bZ5 & $222.4 \pm 16.2$ & $11.6 \pm 0.58$ & $394.9 \pm 187.9$ \\
T3cZ5 & $285.6 \pm 21.6$ & $11.6 \pm 0.67$ & $671.6 \pm 306.3$ \\
T4Z5 & $495.0 \pm 47.7$ & $13.4 \pm 3.55$ & $1196 \pm 643.2$
\end{tabular}

\section{Results}

\subsection{Relative azimuth distribution of horizontal wave vectors}

Since the background wind velocity $(\bar{u}, \bar{v})$ in Eq. (2) of the linear gravity wave theory is presumed to be a constant vector independent of time and height, we take the height average of the 72 (96) h averaged mean wind profile of the winter (summer) data (see Fig. 1) to obtain the constant background wind velocity: $(\bar{u}, \bar{v})=(37.8,8.1) \mathrm{m} / \mathrm{s}$ for the winter data, and $(\bar{u}, \bar{v})=(-8.46,3.08) \mathrm{m} / \mathrm{s}$ for the summer data, or in terms of velocity amplitude $u_{h}$ and its azimuth angle $\phi_{u h}$,
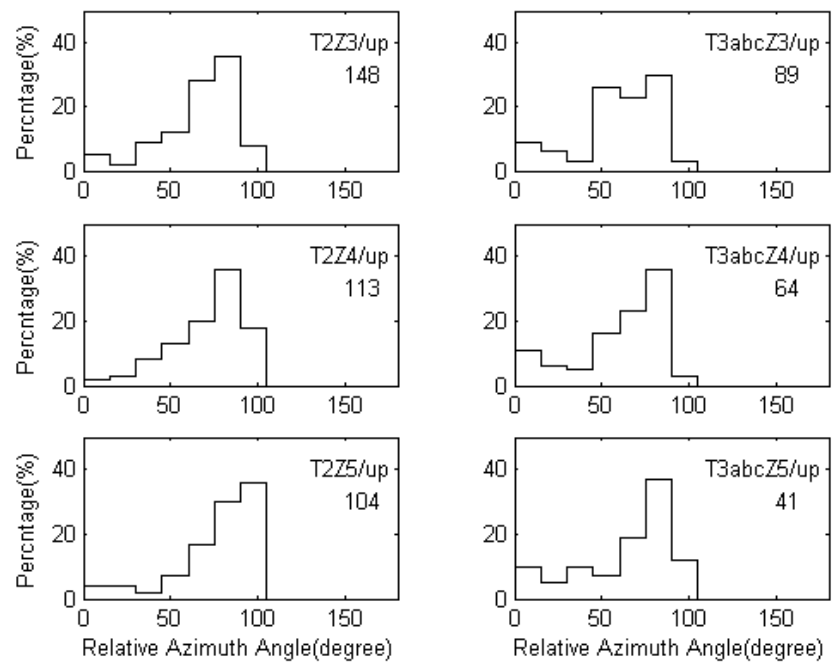

Fig. 2. Distributions of the absolute relative azimuth angles between the characteristic horizontal wave vector of the upward moving gravity wave packets and the mean wind velocity. The letters on the upper-right corner of each diagram denote the window, and the number below it is the number of events identified as AGW packet in that window. The windows were defined in Table 1 . The data was from the winter data set.
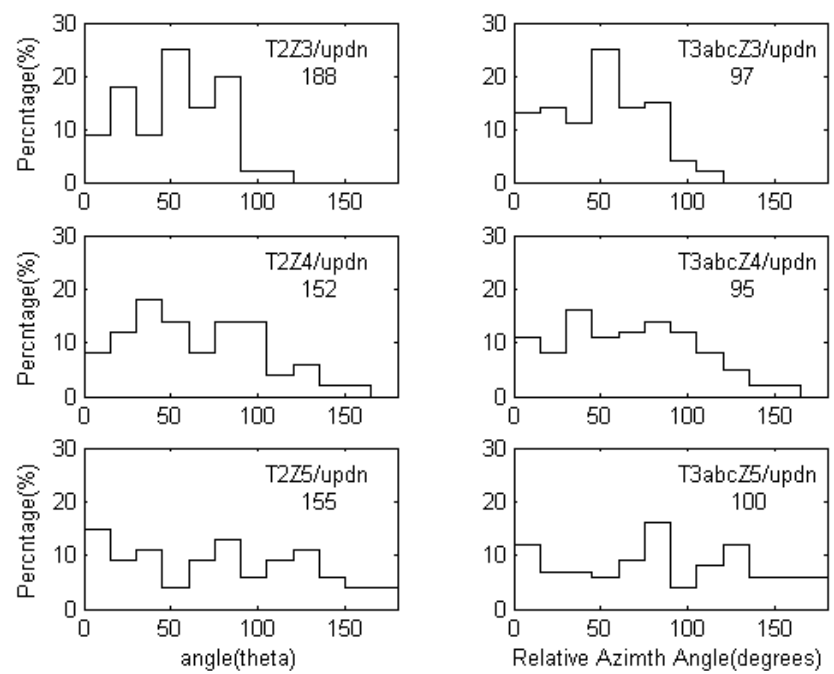

Fig. 3. Same as Fig. 2 except that the data was from the upward and downward moving gravity wave packets of summer data set.

$u_{h}=38.7 \mathrm{~m} / \mathrm{s}, \phi_{u h}=77.9^{\circ}$ for the winter data, and $u_{h}=9 \mathrm{~m} / \mathrm{s}$, $\phi_{u h}=290^{\circ}$ for the summer data. As explained in Sect. 2.2, there are two solution-wave vectors (for dispersion equation) symmetric with respect to the mean wind velocity vector $\boldsymbol{u}_{h}$, and one of them is more likely than the other to be the "virtual wave vector" of the gravity wave packet. Before we decide the virtual wave vector for each wave packet, here we shall study the distribution of the gravity wave packets with respect to their relative azimuth angle $\left|\phi_{k h}-\phi_{u h}\right|$. 
The geometrical meaning of the relative azimuth angle can be demonstrated by the following facts: when the wave vector is in the same direction of the mean wind velocity, we have $\left|\phi_{k h}-\phi_{u h}\right|=0^{\circ}$; when it is in the opposite direction of the mean wind, we have $\left|\phi_{k h}-\phi_{u h}\right|=180^{\circ}$. Figure 2 (Fig. 3) shows the relative azimuth distribution of the upward (upward and downward) gravity wave packets of the winter data (summer data). Some characteristics of these events in Figs. 2 and 3 were listed in Table 4: The characteristic vertical wave lengths were around $4 \sim 6.5 \mathrm{~km}$ for the wave packets in the top panels of Figs. 2 and 3, while that in the middle panels were $7 \sim 10 \mathrm{~km}$, and $10 \sim 13 \mathrm{~km}$ for the packets in the bottom panels; the characteristic wave period of the wave packets in the left panels were around $35 \sim 45 \mathrm{~min}$, while that in the right panels were in the range of $2 \sim 5 \mathrm{~h}$. The characteristics of the distributions are quite different between the winter data and summer data: Fig. 2 shows that the wave vectors of most wave packets of the winter data tended to be perpendicular to the mean wind velocity vector regardless of their wave periods (comparison between left and right panels) and vertical wave lengths (comparison among top, middle and bottom panels). A small percentage of the wave vectors have relative azimuth angles $\left|\phi_{k h}-\phi_{u h}\right|$ in the range $0^{\circ} \sim 50^{\circ}$, and no wave packets were found to have $\left|\phi_{k h}-\phi_{u h}\right|$ in the range $120^{\circ} \sim 180^{\circ}$. Figure 3 shows that the relative azimuth distributions were not different between the shorter period waves (left panels) and longer period waves (right panels), but were strongly dependent on the vertical wavelength. The percentage of the wave vectors pointing against the mean wind increased with increasing vertical wavelength (from top to bottom panels). No wave vectors with short vertical wavelength (top panels) were found to point against the mean wind, while the wave vectors with sufficiently large vertical wavelength (bottom panels), were found to point evenly in all directions.

One common feature demonstrated by Figs. 2 and 3 is that no wave vector with insufficiently large vertical wavelength was detected to point against the mean wind. This feature can be explained by the dispersion Eq. (2): the intrinsic frequency $\omega$ of a wave with fixed wave period and horizontal wave length is maximal when its wave vector points against the mean wind, leading to maximal vertical wavelength. Such a Doppler effect is controlled by the amplitude of the mean wind. Since the winter mean wind $(38.7 \mathrm{~m} / \mathrm{s})$ was much stronger than the summer mean wind $(9 \mathrm{~m} / \mathrm{s})$, the Doppler effect is clearly responsible for the lack of detected wave packets with characteristic wave vectors pointing against the winter mean wind. To confirm this argument, we calculated the vertical wavelengths of the gravity waves (by dispersion equations) with a wave period of $40 \mathrm{~min}$ and different horizontal wavelengths propagating in the winter mean wind $\left(u_{h}=38.7 \mathrm{~m} / \mathrm{s}, \phi_{u h}=77.9^{\circ}\right)$. The results of calculations were listed in Table 5, from which we can see that the vertical wavelengths of the $40 \mathrm{~min}$ gravity waves with $\left|\phi_{k h}-\phi_{u h}\right|=129^{\circ}$ and $\lambda_{h} \sim 144 \mathrm{~km}, 108 \mathrm{~km}$,

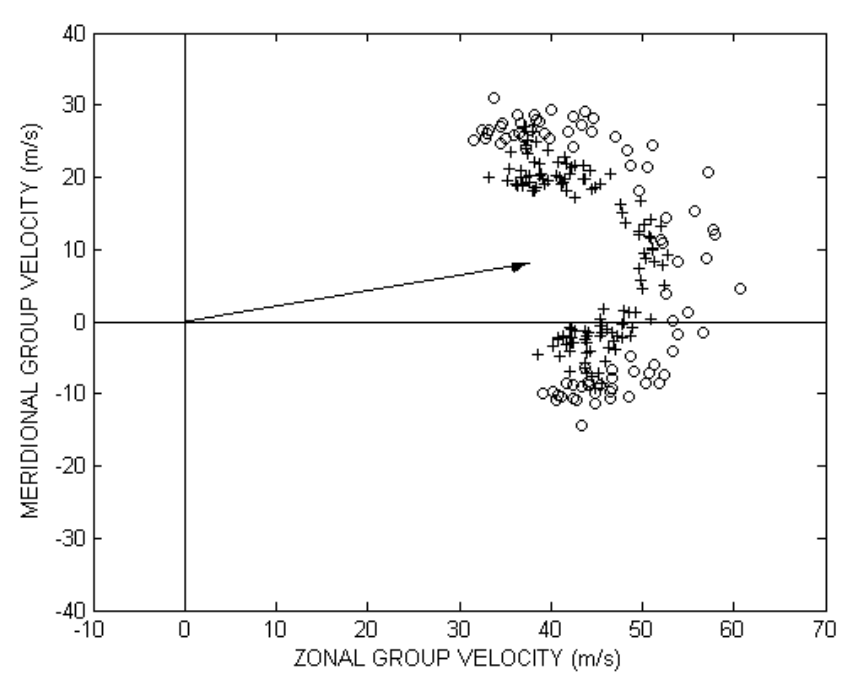

Fig. 4. Scattering plot of the horizontal group velocities of the upward moving gravity wave packets of winter data set. Wave packets obtained by windows T3aZ4, T3bZ4 and T3cZ4 were denoted by cross; wave packets obtained by windows T3aZ5, T3bZ5 and T3cZ5 were denoted by open circle.

$72 \mathrm{~km}, 36 \mathrm{~km}$ (last column) are $64.1 \mathrm{~km}, 50.5 \mathrm{~km}, 39.3 \mathrm{~km}$, $33.0 \mathrm{~km}$, respectively ( $3 \mathrm{rd}$ column). All these waves would be excluded by the windows as listed in Table 1, because their vertical wavelengths were too large. Another interesting feature revealed by Figs. 2 and 3 is that the relative azimuth angles in the summer data was evenly distributed when the vertical wavelengths were sufficiently large (see bottom panels of Fig. 3), and the number of wave packets of winter data increased with increasing relative azimuth angle below a cut-off angle (about 110 degrees, see Fig. 2). Beyond the cutoff angle, the wave packet events (if existed) would be filtered out by the windows due to their larger-thanobservation-range-vertical wave lengths.

\subsection{Virtual horizontal group velocity distribution of gravity wave packets}

Figure 4 is the scattering plot of the horizontal group velocities $\left(\boldsymbol{v}_{g h}\right)$ of the upward moving gravity wave packets, obtained by the windows T3aZ4, T3bZ4, T3cZ4, T3aZ5, T3bZ5 and T3cZ5 of the winter data. Likewise, Fig. 5 is the corresponding scattering plot (also only upward moving gravity wave packets) of the summer data. The arrow in each figure represents the mean wind velocity vector $\boldsymbol{u}_{h}$. The scattering plot of the group velocities $\boldsymbol{v}_{g h}$ of the winter data (Fig. 4) seemingly scattered around a semicircle with its axis perpendicular to the mean wind vector $\boldsymbol{u}_{h}$ and with its center at the tip of $\boldsymbol{u}_{h}$. It clearly demonstrated the dominant contribution of the strong mean wind to the horizontal group velocities of the gravity wave packets. The corresponding scattering plot of the summer data (Fig. 5) nearly formed a circle with its center at the tip of the mean wind velocity 
Table 5. Characteristic physical parameters $\left(v_{p z}, v_{g z}, \lambda_{z}, \tau_{i}, v_{p h}, \phi_{k h}, \lambda_{h}\right)$ of the AGW wave packets with a characteristic wave period of $\tau=40 \mathrm{~min}$ obtained by dispersion relation under the winter background wind with $u_{E}=37.8 \mathrm{~m} / \mathrm{s}$ and $u_{N}=8.1 \mathrm{~m} / \mathrm{s}\left(u_{h}=38.7 \mathrm{~m} / \mathrm{s}\right.$, $\left.\phi_{u h}=77.9^{\circ}\right) . \phi_{k h}$ and $\phi_{u h}$ are the azimuth angles of the horizontal wave vector and mean wind velocity, respectively.

\begin{tabular}{ccccccc}
\hline$v_{p z}(\mathrm{~m} / \mathrm{s})$ & $v_{g z}(\mathrm{~m} / \mathrm{s})$ & $\lambda_{z}(\mathrm{~km})$ & $\tau_{i}(\mathrm{~h})$ & $v_{p h}(\mathrm{~m} / \mathrm{s})$ & $\left|\phi_{k h}-\phi_{u h}\right|\left(^{\circ}\right)$ & $\lambda_{h}(\mathrm{~km})$ \\
\hline $\mathbf{- 7 . 6 7}$ & $\mathbf{3 . 6 4}$ & $\mathbf{1 8 . 4}$ & $\mathbf{1 . 3 3}$ & $\mathbf{6 0}$ & $\mathbf{3 9}$ & $\mathbf{1 4 4}$ \\
-16.5 & 13.2 & 39.5 & 0.67 & 60 & 89 & 144 \\
-26.7 & 23.4 & 64.1 & 0.47 & 60 & 129 & 144 \\
-35.4 & 28.2 & 85.0 & 0.41 & 60 & 179 & 144 \\
$-\mathbf{3 . 7 6}$ & $\mathbf{1 . 2 2}$ & $\mathbf{9 . 0}$ & $\mathbf{2 . 0}$ & $\mathbf{4 5}$ & $\mathbf{3 9}$ & $\mathbf{1 0 8}$ \\
-11.9 & 10.2 & 28.6 & 0.68 & 45 & 89 & 108 \\
-21.0 & 22.0 & 50.5 & 0.43 & 45 & 129 & 108 \\
-28.6 & 28.4 & 68.6 & 0.36 & 45 & 179 & 108 \\
$-\mathbf{0 . 9 2}$ & $\mathbf{- 0 . 1 1}$ & $\mathbf{2 . 2}$ & $-\mathbf{5 . 2 9}$ & $\mathbf{3 0}$ & $\mathbf{2 9}$ & $\mathbf{7 2}$ \\
-7.7 & 6.85 & 18.5 & 0.68 & 30 & 89 & 72 \\
-16.4 & 20.5 & 39.3 & 0.37 & 30 & 129 & 72 \\
-24.2 & 27.9 & 58.0 & 0.29 & 30 & 179 & 72 \\
$-\mathbf{3 . 8 9}$ & $-\mathbf{3 . 6 1}$ & $\mathbf{9 . 3}$ & $-\mathbf{0 . 6 7}$ & $\mathbf{1 5}$ & $\mathbf{3 9}$ & $\mathbf{3 6}$ \\
-3.72 & 3.33 & 8.9 & 0.69 & 15 & 89 & 36 \\
-13.7 & 18.5 & 33.0 & 0.25 & 15 & 129 & 36 \\
-42.9 & 15.0 & 102.9 & 0.19 & 15 & 179 & 36 \\
$-\mathbf{1 0 . 4}$ & $-\mathbf{1 0 . 5}$ & $\mathbf{2 4 . 9}$ & $\mathbf{- 0 . 2}$ & $\mathbf{7}$ & $\mathbf{3 9}$ & $\mathbf{1 6 . 8}$ \\
-1.64 & 1.42 & 3.9 & 0.73 & 7 & 89 & 16.8 \\
-18.1 & 8.48 & 43.4 & 0.18 & 7 & 119 & 16.8 \\
\hline
\end{tabular}

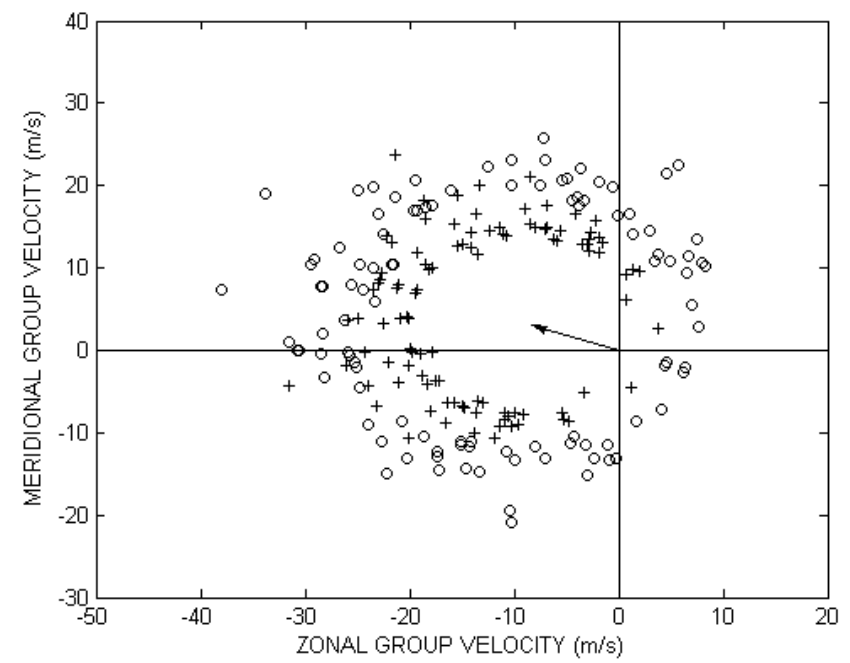

Fig. 5. Same as Fig. 4 except that the data were from the upward moving gravity wave packets of the summer data set.

vector. This contrast between these two plots was evidently resulted from a very strong mean wind in winter and a rather weak mean wind in summer.

Because the dispersion equation has two symmetric solutions (with respect to the mean wind) for each wave packet measurement, each plot consists of two symmetric halves separated by the axis extending along the mean wind velocity vector. But at most one of the two solutions can be

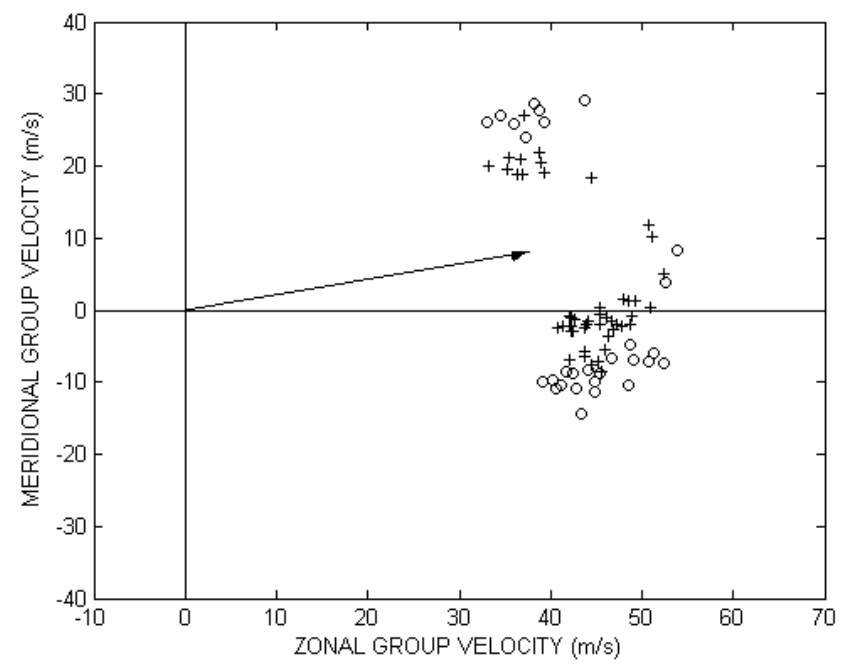

Fig. 6. Scattering plot of the virtual horizontal group velocities of the upward gravity wave packets of the winter data. Wave packets obtained by windows T3aZ4, T3bZ4 and T3cZ4 were denoted by cross; wave packets obtained by windows T3aZ5, T3bZ5 and T3cZ5 were denoted by open circle. These gravity wave packets were selected from the gravity wave packets in Fig. 4.

real, so only part of Figs. 4 and 5 can represent the "virtual group velocity distributions". We need one more parameter to decide the true solution, and a possible parameter is the vertical flux of horizontal momentum carried by the gravity 


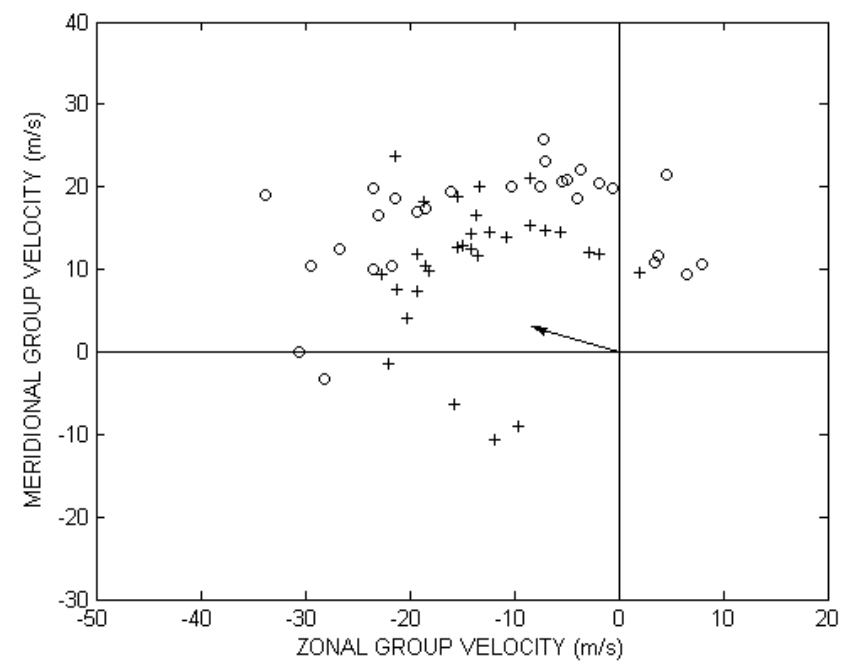

Fig. 7. Same as Fig. 6 except that these gravity wave packets were selected from the the summer data set in Fig. 5.

wave packet. Under the assumption that the waves propagate without dissipation through a background atmosphere (that varies only in the vertical), the vertical flux of horizontal pseudo-momentum

$$
\left(\Im_{x z}, \Im_{y z}\right)=\rho_{0} v_{g z} \frac{E}{\omega}(k, \ell)
$$

is related to the Reynolds stress and vertical flux of horizontal momentum as (Fritts and Alexander, 2003)

$$
\left(\Im_{x z}, \Im_{y z}\right)=\rho_{0}\left(1-f^{2} / \omega^{2}\right)\left(\overline{u^{\prime} w^{\prime}}, \overline{v^{\prime} w^{\prime}}\right),
$$

where

$$
E=\overline{u^{\prime} u^{\prime}}+\overline{v^{\prime} v^{\prime}}
$$

and $\rho_{0}$ is the mass density of the atmosphere. We proposed that the wave packet-related averaging quantities $\overline{u^{\prime} u^{\prime}}+\overline{v^{\prime} v^{\prime}}$, $\overline{u^{\prime} w^{\prime}}$, and $\overline{v^{\prime} w^{\prime}}$ could be calculated within a box of time range $T$ and height range $Z$, with its center coinciding with the center of the corresponding wave packet. If the scale of the box $(T, Z)$ was changed, these averaging values will also change correspondingly. Since these averages are statistical quantities, they can be used only as a reference to decide which one, between the two symmetric solutions of the dispersion equation, is more likely to be the true solution. In this study, we calculated the averages over four boxes with scale lengths $\left(\tau, \lambda_{z}\right),\left(\tau, \lambda_{z} / 2\right),\left(\tau, \lambda_{z} / 4\right)$, and $\left(\tau, \lambda_{z} / 8\right)$, where $\tau$ and $\lambda_{z}$ are the characteristic wave period and vertical wavelength respectively of the corresponding wave packet. For each calculation, the values of the averages were substituted into Eqs. (7) and (8) to obtain a horizontal reference-wavevector $\left(k^{\prime}, \ell^{\prime}\right)$. Then we picked the solution-wave-vector $(k, \ell)$ which is closer than the other to $\left(k^{\prime}, \ell^{\prime}\right)$ as the "virtual

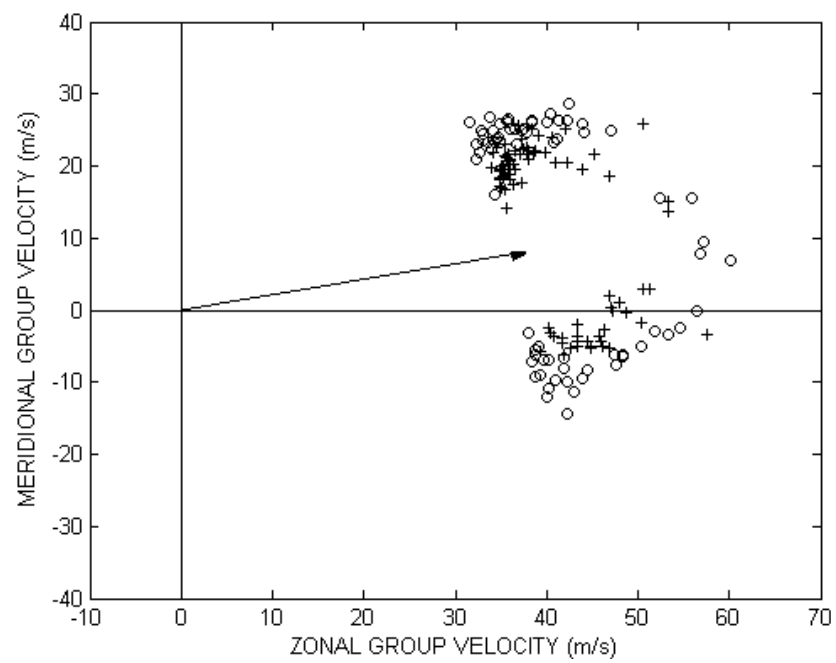

Fig. 8. Scattering plot of the virtual horizontal group velocities of the upward moving gravity wave packets of the winter data set. Wave packets obtained by window T2Z4 were denoted by cross; wave packets obtained by window T2Z5 were denoted by open circle.

wave vector" of the gravity wave packet if it also satisfies following condition:

$0.33<k_{h} / k_{h}^{\prime}<3 \quad$ and $\quad\left|\phi_{k h}-\phi_{k h}^{\prime}\right|<90^{\circ}$.

Here, $k_{h}^{\prime}$ and $\phi_{k h}^{\prime}$ are the amplitude and the azimuth angle of the reference-wave-vector, respectively. If such a condition (10) is not satisfied for each calculation, then the corresponding gravity wave packet would be removed. This selection criteria was based on the result of our numerical study of Eqs. (7) and (8) (see Appendix B) and the consideration of the possible measurement error and the effect of multiplewave-superposition. Our numerical results showed that for a monochromatic wave, the differences of the azimuths of the horizontal pseudo-momentum in Eq. (7) and momentum in Eq. (8) were less than $3^{\circ}$ (see Fig. B1); and the ratios between their amplitudes was between 0.5 and 2.0 (see Fig. B2). For the case of multiple-wave-superposition such as the case of wave packet, the differences will be larger. By this selection process, 77 out of 105 gravity wave packets $(73 \%)$ in Fig. 4 and 60 out of 105 gravity wave packets (57\%) in Fig. 5 were found to meet the condition (10), and the scattering plot of the "virtual horizontal group velocities" of the gravity wave packets in Fig. 6 (reduced from winter events in Fig. 4) and Fig. 7 (reduced from summer events in Fig. 5) were obtained.

The virtual horizontal group velocities of the gravity wave packets with periods in the range $2 \sim 5 \mathrm{~h}$ of the winter data (Fig. 6) were dominantly in the direction of east-south-east. There were also a significant number of wave packets propagating in the north-east direction. The corresponding gravity wave packets of the summer data (Fig. 7) tended to propagate uniformly in the section between west-north-west and north 


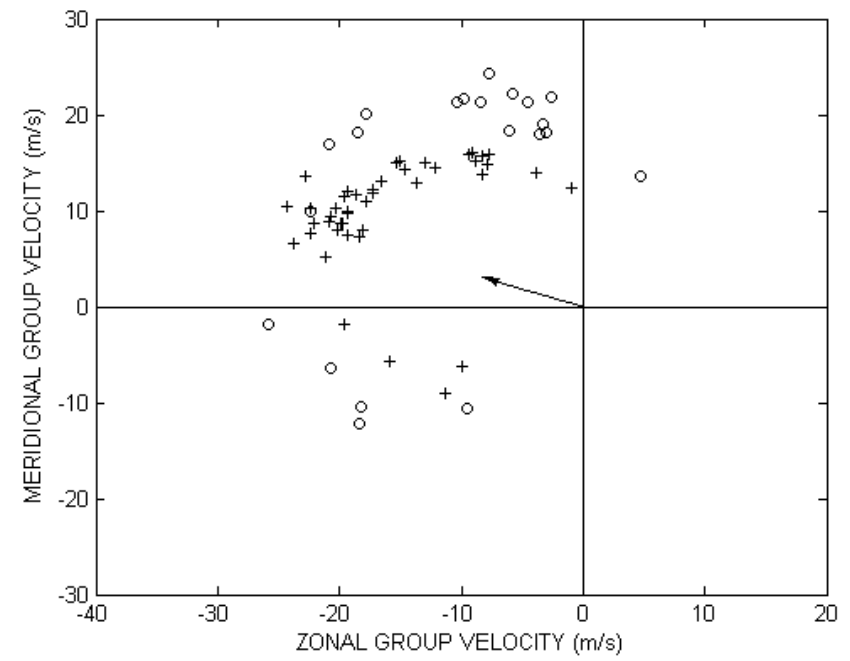

Fig. 9. Same as Fig. 8 except that the wave packets were from summer data set.

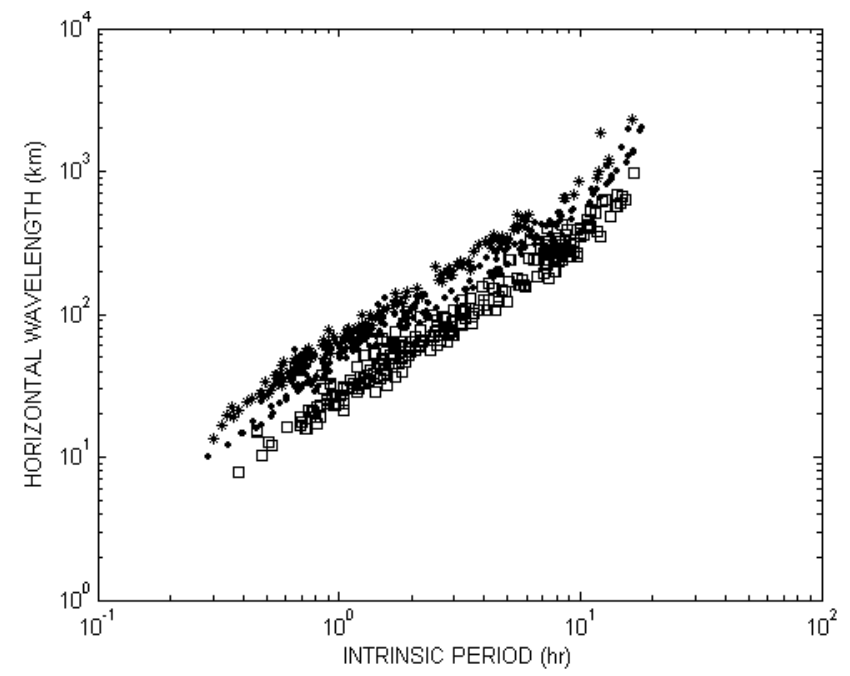

Fig. 10. Horizontal wavelengths versus intrinsic periods of upward moving gravity wave packets of winter data set. Wave packets obtained by window T2Z3, T3aZ3, T3bZ3, T3cZ3 and T4Z3 were denoted by square; Wave packets obtained by window T2Z4, T3aZ4, T3bZ4, T3cZ4 and T4Z4 were denoted by dot; Wave packets obtained by window T2Z5, T3aZ5, T3bZ5, T3cZ5 and T4Z5 were denoted by star.

directions. The same procedures were also applied to the upward gravity wave packets obtained from the windows T2Z4 and T2Z5 (with wave periods in the range $30 \mathrm{~min} \sim 1 \mathrm{~h}$ ) for both winter data and summer data. The corresponding scattering plots of the virtual horizontal group velocities were shown in Fig. 8 (winter data) and Fig. 9 (summer data). Again, the gravity wave packets of the summer data (Fig. 9) tended to propagate uniformly in the section between westnorth-west and north directions, but the winter data (Fig. 8)

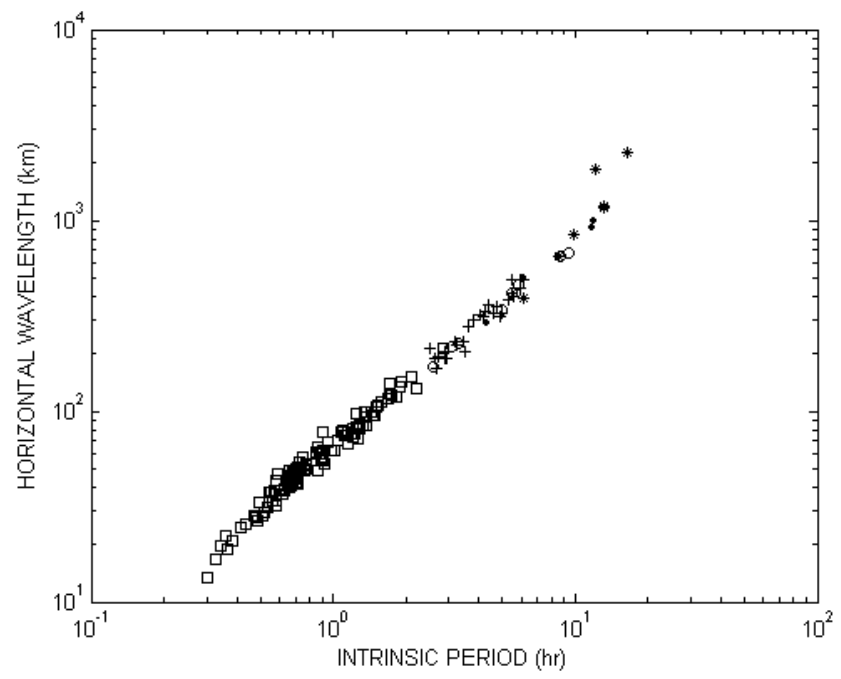

Fig. 11. Horizontal wavelengths versus intrinsic periods of upward moving gravity wave packets of winter data set. Wave packets obtained by window T2Z5 were denoted by square; Wave packets obtained by window T3aZ5 were denoted by cross; Wave packets obtained by window T3bZ5 were denoted by open circle; Wave packets obtained by window T3cZ5 were denoted by dot'; Wave packets obtained by window T4Z5 were denoted by star.

shows that the north-eastward gravity wave packets were slightly more than the east-south-eastward ones.

\subsection{Positive correlation between horizontal wavelength and intrinsic period}

Figure 10 shows a clear positive correlation between the horizontal wavelength and the intrinsic period of the upward gravity wave packets of the winter data, obtained from three groups of windows: (1) T2Z5, T3aZ5, T3bZ5, T3cZ5, T4Z5; (2) T2Z4, T3aZ4, T3bZ4, T3cZ4, T4Z4; (3) T2Z3, T3aZ3, T3bZ3, T3cZ3, T4Z3. The characteristic vertical wave lengths were around $10 \sim 13 \mathrm{~km}$ for the gravity wave packets in group (1), while $7 \sim 10 \mathrm{~km}$ for the packets in group (2), and $4 \sim 6.5 \mathrm{~km}$ for the packets in group (3). The characteristic (observed) wave periods covered a range from 0.5 to $8 \mathrm{~h}$. There were three separate straight lines formed by the wave packets of three different groups (different vertical wavelength ranges) in Fig. 10, where type 2 wave packets (negative intrinsic period) were not included. The horizontal wavelength $\lambda_{h}$ of the wave packets in group (1), as shown independently in Fig. 11, has a relationship with intrinsic pe$\operatorname{riod} \tau_{i}$ as

$\lambda_{h}=0.72 \tau_{i}^{1.10}(r=0.99)$;

And the corresponding relationships for the wave packets of group (2) and group (3) were fitted by Eqs. (12) and (13), respectively,

$\lambda_{h}=0.50 \tau_{i}^{1.11}(r=0.99)$; 


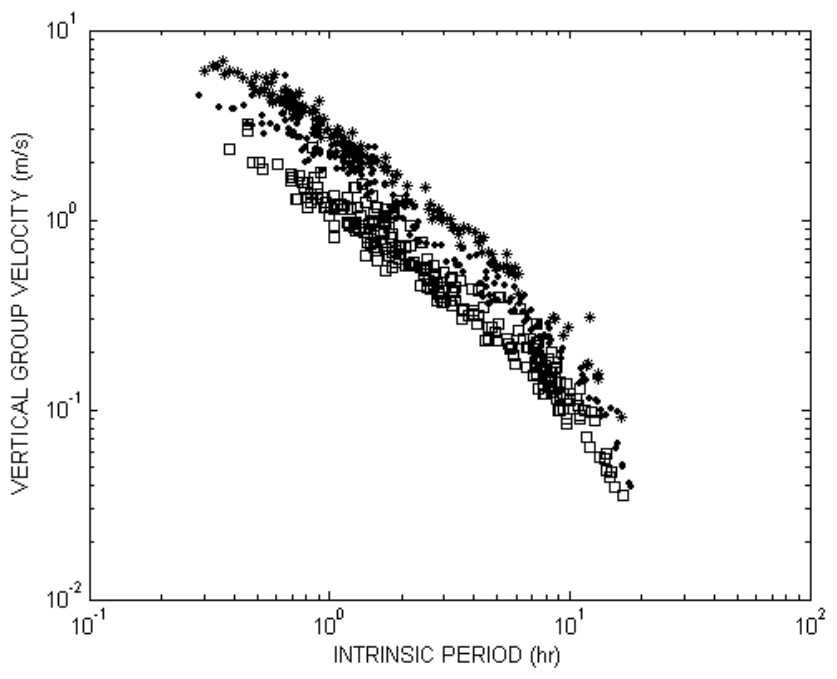

Fig. 12. Vertical group velocities versus intrinsic periods of upward moving gravity wave packets of winter data set. Wave packets obtained by window T2Z3, T3aZ3, T3bZ3, T3cZ3 and T4Z3 were denoted by square; Wave packets obtained by window T2Z4, T3aZ4, T3bZ4, T3cZ4 and T4Z4 were denoted by dot; Wave packets obtained by window T2Z5, T3aZ5, T3bZ5, T3cZ5 and T4Z5 were denoted by star.

$\lambda_{h}=0.29 \tau_{i}^{1.11}(r=0.98)$,

where $r$ represents the correlation coefficient. The corresponding results for the summer data (including both upward and downward wave packets) were given in Eqs. (14), (15) and (16) for the wave packets of group (1), (2) and (3), respectively,

$\lambda_{h}=0.88 \tau_{i}^{1.05}(r=0.99)$;

$\lambda_{h}=0.61 \tau_{i}^{1.06}(r=0.99)$;

$\lambda_{h}=0.31 \tau_{i}^{1.08}(r=0.98)$.

Clearly, the spreading of the distribution in Fig. 10 resulted from the spreading of the vertical wavelengths.

\subsection{Negative correlation between vertical group velocity and intrinsic period}

In contrast to the positive correlation between $\lambda_{h}$ and $\tau_{i}$, the correlation between the vertical group velocity $v_{g z}$ and intrinsic period $\tau_{i}$ is negative as shown in Fig. 12, where the wave packets were the same as those of Fig. 10. The relationships between $v_{g z}$ and $\tau_{i}$ for the upward gravity wave packets of winter data in group (1), (2) and (3) were fitted by Eqs. (17), (18), and (19), respectively:

$v_{g z}=165.8 \tau_{i}^{-0.99}(r=-0.99)$,

$v_{g z}=195.6 \tau_{i}^{-1.09}(r=-0.98)$, $v_{g z}=102.8 \tau_{i}^{-1.06}(r=-0.98)$.

Similarly, the corresponding relationships for the wave packets (upward and downward packets together) of summer data in group (1), (2), and (3) were fitted by Eqs. (20), (21), and (22), respectively:

$\left|v_{g z}\right|=163.7 \tau_{i}^{-0.99}(r=-0.99)$,

$\left|v_{g z}\right|=131.9 \tau_{i}^{-1.01}(r=-0.99)$,

$\left|v_{g z}\right|=93.2 \tau_{i}^{-1.06}(r=-0.98)$.

Again, the spreading of the distribution in Fig. 12 also resulted from the spreading of the vertical wavelengths. The power law relations in Eqs. (11 22) were approximate results of the dispersion equation, but they offer simpler formulas to describe the relations among the physical parameters of AGWs.

\subsection{Statistical error of propagation parameters due to uncertainty of BV-frequency}

The calculations of the characteristic propagation parameters of wave packets depend on the measurement values of $v_{p z}, v_{g z}$ and $\tau$, and the environmental parameters $N, f$ and $(\bar{u}, \bar{v})$. In addition to the measurement errors in $v_{p z}$, $v_{g z}$, and $\tau$, there is a systematic error induced by the uncertainty of the value of the BV-frequency $N$. The results presented in Sect. 3 were based on the assumption of $N=1.047 \times 10^{-2} \mathrm{~s}^{-1}$ (10 min BV-period). Here we shall present some calculations on the winter wave packets using another extreme value of $N=2.094 \times 10^{-2} \mathrm{~s}^{-1}$ ( $5 \mathrm{~min} \mathrm{BV}$ period). Table 6a presented the statistics (mean \pm standard deviation) of the calculated values of the characteristic intrinsic period $\tau_{i}$, horizontal wavelength $\lambda_{h}$, and the azimuth angle $\varphi_{k h}$ of the horizontal wave vector using $N=2.094 \times 10^{-2} \mathrm{~s}^{-1}$ (5 min BV-period). The corresponding statistics using $N=1.047 \times 10^{-2} \mathrm{~s}^{-1}(10 \mathrm{~min} \mathrm{BV}$-period) was listed in Table $6 \mathrm{~b}$ for comparison. It is readily noted that when the $\mathrm{BV}$-period is changed from $10 \mathrm{~min}$ to $5 \mathrm{~min}$, the calculated intrinsic periods become significantly smaller, while the horizontal wavelengths become significantly larger, and the azimuth angles change by about 10 degrees tending toward the direction of the background wind velocity as can be seen also from Fig. 13. The comparison between the results of these two model calculations of horizontal wavelength versus intrinsic period were demonstrated in Fig. 14, where the results of model calculation using $N=1.047 \times 10^{-2} \mathrm{~s}^{-1}(10 \mathrm{~min} \mathrm{BV}$ period) were presented by black symbols, while that using $N=2.094 \times 10^{-2} \mathrm{~s}^{-1}$ (5 min BV-period) were presented by red symbols. Comparison of the scattering plots of the virtual horizontal group velocities of the upward gravity wave packets of the winter data set from windows T3aZ4, T3bZ4, T3cZ4, T3aZ5, T3bZ5 and T3cZ5 were presented in Fig. 15, and the corresponding scattering plot from windows T2Z4 
Table 6a. Mean \pm standard deviation of characteristic intrinsic wave period $\tau_{i}$, horizontal wavelength $\lambda_{h}$, and azimuth angle of horizontal wave vector $\varphi_{k h}$ of the atmospheric gravity wave packets with upward group velocity. These parameters were obtained by dispersion equations assuming BV-period equal to $5 \mathrm{~min}$. The events were from winter data set. Note that the two values of $\varphi_{k h}$ are symmetric with respect to the azimuth angle $\left(77.9^{\circ}\right)$ of the mean wind velocity vector.

\begin{tabular}{cccc}
\hline Window & $\tau_{i}(\mathrm{~h})$ & $\lambda_{h}(\mathrm{~km})$ & $\varphi_{k h}\left(^{\circ}\right)$ \\
\hline T2Z3 & $1.434 \pm 0.568$ & $84.26 \pm 36.55$ & $19.2 \pm 27.1$ \\
& & & $136.6 \pm 27.1$ \\
T2Z4 & $1.056 \pm 0.369$ & $106.3 \pm 42.05$ & $12.8 \pm 27.5$ \\
& & & $143.0 \pm 27.5$ \\
T2Z5 & $0.853 \pm 0.299$ & $113.2 \pm 41.91$ & $3.0 \pm 29.5$ \\
& & & $152.8 \pm 29.5$ \\
T3abcZ3 & $6.834 \pm 1.894$ & $459.8 \pm 171.5$ & $30.2 \pm 28.3$ \\
& & & $125.6 \pm 28.3$ \\
T3abcZ4 & $5.632 \pm 1.919$ & $585.9 \pm 238.0$ & $27.3 \pm 27.5$ \\
& & & $128.5 \pm 27.5$ \\
T3abcZ5 & $3.779 \pm 1.124$ & $541.6 \pm 183.1$ & $14.0 \pm 30.2$ \\
& & & $141.8 \pm 30.2$ \\
\hline
\end{tabular}

Table 6b. Same as Table 6a except that the BV-period was assumed to be $10 \mathrm{~min}$.

\begin{tabular}{cccc}
\hline Window & $\tau_{i}(\mathrm{~h})$ & $\lambda_{h}(\mathrm{~km})$ & $\varphi_{k h}\left(^{\circ}\right)$ \\
\hline T2Z3 & $1.792 \pm 0.872$ & $53.92 \pm 28.45$ & $10.8 \pm 21.4$ \\
& & & $145.0 \pm 21.4$ \\
T2Z4 & $1.233 \pm 0.550$ & $62.71 \pm 30.59$ & $6.7 \pm 21.2$ \\
& & & $149.1 \pm 21.2$ \\
T2Z5 & $0.951 \pm 0.461$ & $63.30 \pm 33.82$ & $0.4 \pm 25.1$ \\
& & & $155.4 \pm 25.1$ \\
T3abcZ3 & $7.914 \pm 2.515$ & $283.8 \pm 123.6$ & $18.6 \pm 24.8$ \\
& & & $137.2 \pm 24.8$ \\
T3abcZ4 & $6.507 \pm 2.476$ & $359.5 \pm 180.2$ & $17.7 \pm 24.9$ \\
& & & $138.1 \pm 24.9$ \\
T3abcZ5 & $4.809 \pm 2.209$ & $357.7 \pm 182.5$ & $13.7 \pm 26.9$ \\
& & & $142.1 \pm 26.9$ \\
\hline
\end{tabular}

and T2Z5 were presented in Fig. 16. We can roughly claim from Figs. 15 and 16 that the statistics of the propagation direction of the wave packets are not much different between these two model calculations.

\section{Summaries and discussions}

It is universally accepted that the wave energy and momentum are transported at its group velocity. So the direction of vertical group velocity of the AGW has been unanimously regarded as the vertical direction of the wave energy and momentum transport. However, the horizontal group velocity of
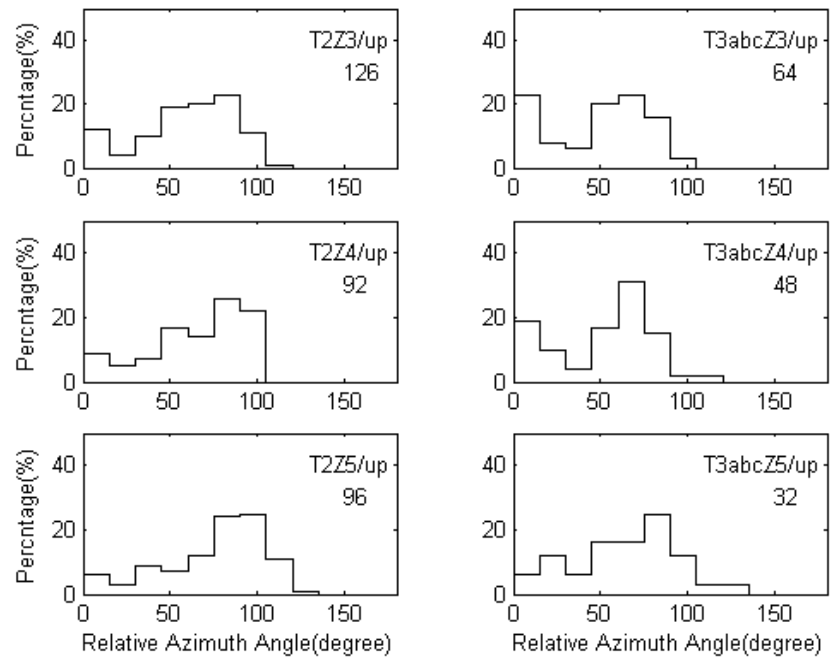

Fig. 13. Same as Fig. 2 except that the VB-period of 5 min was applied in this calculation.

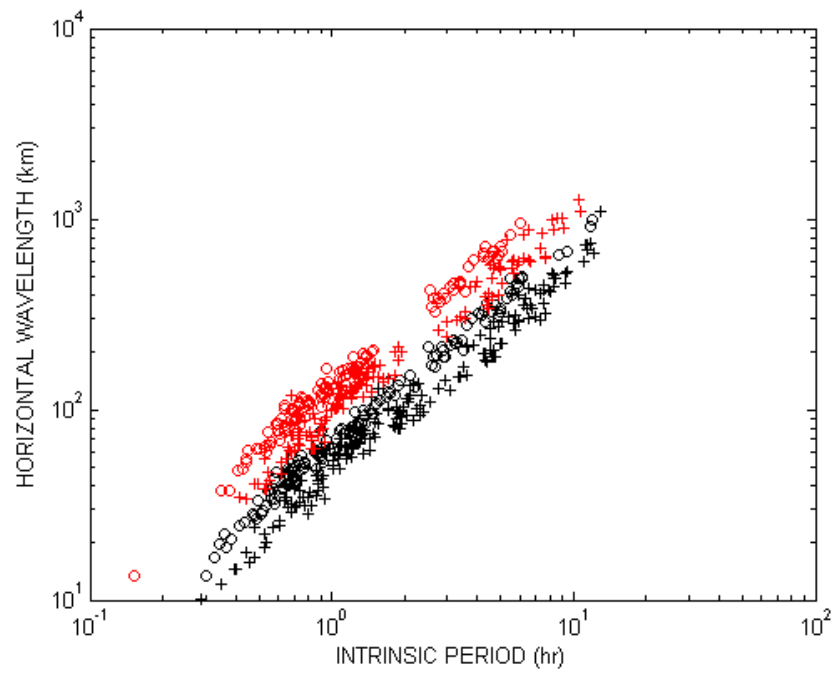

Fig. 14. Horizontal wavelength versus intrinsic period of upward moving gravity wave packets of winter data set. Wave packets obtained by window T2Z4, T3aZ4, T3bZ4, T3cZ4 and T4Z4 were denoted by cross; Wave packets obtained by window T2Z5, T3aZ5, T3bZ5, T3cZ5 and T4Z5 were denoted by open circle. Red colour symbols represent the model with VB-period of $5 \mathrm{~min}$, and black colour symbols represent the model of VB-period of $10 \mathrm{~min}$.

AGW has not been equally treated. The direction of the horizontal wave vector (the direction of horizontal phase velocity) had been regarded as the direction of wave propagation in most of the existing literature, which is alright only when the background wind is negligible, because then the direction of the horizontal group velocity of the AGW is the same as that of its horizontal wave vector. When the background wind is not negligible, such as the case of the winter data in this study, the azimuth angle of the horizontal wave vector 


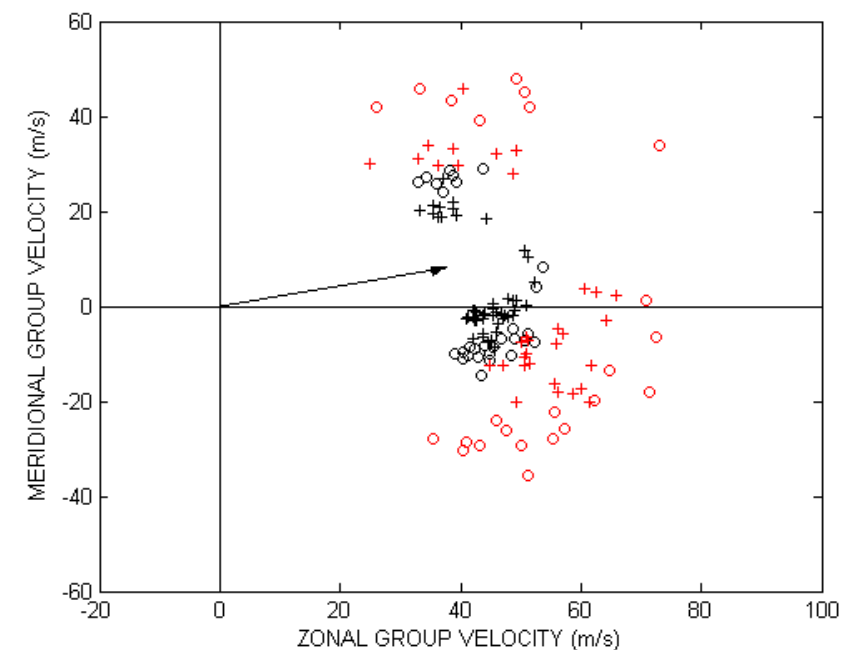

Fig. 15. Scattering plots of the virtual horizontal group velocities of the upward gravity wave packets of the winter data. Wave packets obtained by windows T3aZ4, T3bZ4 and T3cZ4 were denoted by cross; wave packets obtained by windows T3aZ5, T3bZ5 and T3cZ5 were denoted by open circle. Red colour symbols represent the model with VB-period of 5 min, and black colour symbols represent the model of VB-period of $10 \mathrm{~min}$.

no longer equals to the azimuth angle of the horizontal group velocity (see Appendix B), we must take horizontal group velocity into consideration.

Phase-and-group-velocity-tracing technique had been proved to be efficient to estimate the vertical group-andphase-velocities $\left(v_{g z}\right.$ and $\left.v_{p z}\right)$ as well as the period $\tau$ of a wave packet (Kuo et al., 2003, 2007, 2008). In this study, we further proposed a process to calculate the horizontal group velocity $\boldsymbol{v}_{g h}$ from the dispersion equations using the measured values of $v_{g z}, v_{p z}$, and $\tau$. There were two issues in this process: first, since the constant background wind velocity $\boldsymbol{u}_{h}$ in the dispersion Eq. (2) is presumed to be independent of time and height, we proposed that this constant can be approximated by taking the time- and height-average of the wind field to remove its fluctuation component. Then how to properly choose the time range and height range for the averaging process may become the issue for future discussion. Second, Eq. (2) has two solutions for the horizontal wave vector $\boldsymbol{k}_{h}$ mathematically, but only one of them is the virtual solution physically. To select the virtual wave vector from the two mathematical-solution-wave vectors, we proposed to use the vertical flux of horizontal momentum as a reference for the wave vector to determine its direction. We notice that the wave vector obtained from the dispersion equation is deterministic, while the vertical flux of horizontal momentum obtained by the averaging process is statistical. Then it is rather natural that the reference wave vector $\left(k^{\prime}, \ell^{\prime}\right)$ inferred from the vertical flux estimation is not consistent with the true horizontal wave vector $(k, \ell)$. Our assumption is simply that between the two solution-wave-vectors, the one which is

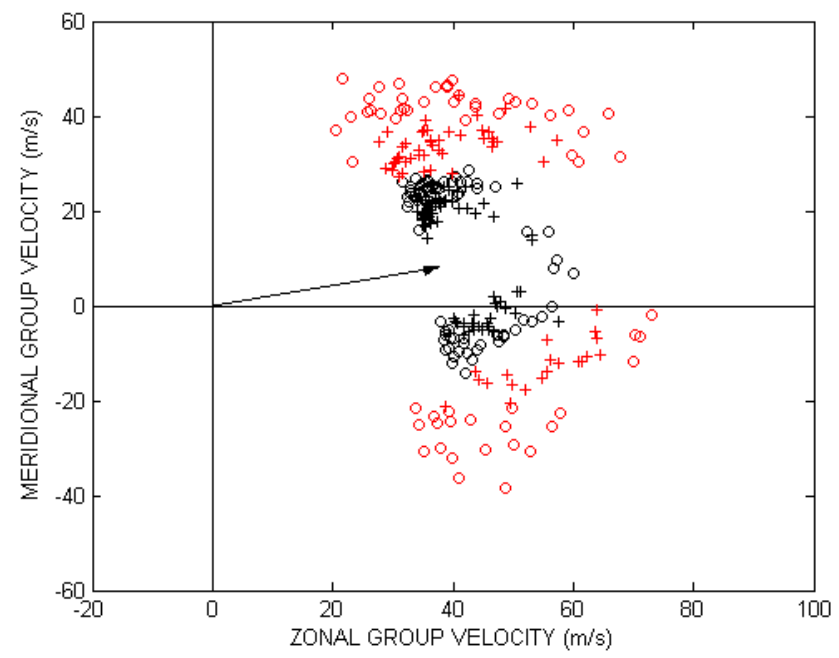

Fig. 16. Scattering plots of the virtual horizontal group velocities of the upward gravity wave packets of the winter data. Wave packets obtained by windows T2Z4 were denoted by cross; wave packets obtained by windows T2Z5 were denoted by open circle. Red colour symbols represent the model with VB-period of $5 \mathrm{~min}$, and black colour symbols represent the model of VB-period of $10 \mathrm{~min}$.

closer to the reference wave vector is more likely to be the virtual wave vector.

The windows used in this study had limited the periods of the wave packets under analysis to be in the range $30 \mathrm{~min} \sim 10 \mathrm{~h}$, and the vertical wavelengths in the range $4 \mathrm{~km} \sim 15 \mathrm{~km}$. Their corresponding horizontal wavelengths were found to be between $20 \mathrm{~km}$ and $1500 \mathrm{~km}$, the intrinsic wave periods were between $18 \mathrm{~min}$ and $20 \mathrm{~h}$, and the horizontal phase velocities were in the range of $15 \sim 60 \mathrm{~m} / \mathrm{s}$. The characteristic vertical wavelength $\lambda_{z}$ of the detectable wave packets was upper-limited by the observation range. An AGW with its horizontal wave vector $\boldsymbol{k}_{h}$ opposite to the mean wind velocity $\boldsymbol{u}_{h}$ would have larger $\lambda_{z}$ than that of the same AGW with its $\boldsymbol{k}_{h}$ parallel to $\boldsymbol{u}_{h}$. Consequently, all the wave packets of the winter data with their $\boldsymbol{k}_{h}$ opposite to $\boldsymbol{u}_{h}$ were not observable, and the group velocities of the observable winter AGWs were concentrated in the directions of east-south-east and north-east. In the case of summer data, the mean wind was much weaker than the winter mean wind, a limited number of wave packets with $\boldsymbol{k}_{h}$ opposite to $\boldsymbol{u}_{h}$ were detectable, and the group velocities of most of the AGW packets were found to be concentrated in the section between west-north-west and the north direction. 


\section{Appendix A}

\section{Procedure of wave packet analysis of phase and group velocity}

\section{A1 Separation of upward waves and downward waves}

Consider an oscillation profile of the meridional (zonal) wind velocity of atmospheric motion $\psi(z, t)$ as a function of height $z$ and time $t$. First we make finite Fourier transformation of $\psi(z, t)$ over the height coordinate $z$ at each fixed time $t$ to obtain the coefficients $A_{n}(t)$ and $B_{n}(t)$ of the function $\cos (n z)$ and the function $\sin (n z)$ respectively:

$\psi(z, t)=\sum_{n}\left\{A_{n}(t) \cdot \cos (n z)+B_{n}(t) \cdot \sin (n z)\right\}$

Then, finite Fourier transformations are made on each $A_{n}(t)$ and $B_{n}(t)$ over time $t$ :

$$
\begin{aligned}
& A_{n}(t)=\sum_{\sigma}\left\{A_{n \sigma}^{(1)} \cdot \cos (\sigma \cdot t)+B_{n \sigma}^{(1)} \cdot \sin (\sigma \cdot t)\right\} \\
& B_{n}(t)=\sum_{\sigma}\left\{A_{n \sigma}^{(2)} \cdot \cos (\sigma \cdot t)+B_{n \sigma}^{(2)} \cdot \sin (\sigma \cdot t)\right\},
\end{aligned}
$$

where $n$ and $\sigma$ represent the vertical wave-number and the observed frequency of the component wave, respectively. Substituting Eqs. (A2a) and (A2b) back into Eq. (A1) with some algebra manipulations, we obtain

$$
\begin{aligned}
\psi(z, t)= & \sum_{n, \sigma} \frac{1}{2}\left\{\left(A_{n \sigma}^{(1)}+B_{n \sigma}^{(2)}\right) \cdot \cos (\sigma \cdot t-n z)\right. \\
& \left.+\left(B_{n \sigma}^{(1)}-A_{n \sigma}^{(2)}\right) \cdot \sin (\sigma \cdot t-n z)\right\} \\
& +\sum_{n, \sigma} \frac{1}{2}\left\{\left(A_{n \sigma}^{(1)}-B_{n \sigma}^{(2)}\right) \cdot \cos (\sigma \cdot t+n z)\right. \\
& \left.+\left(B_{n \sigma}^{(1)}+A_{n \sigma}^{(2)}\right) \cdot \sin (\sigma \cdot t+n z)\right\}
\end{aligned}
$$

The first term in Eq. (A3) represents the contributions from all the waves with upward phase velocities, and the second term comes from the contributions of all the waves with downward phase velocities. Thus, the original oscillation profile $\psi(z, t)$ can be divided into phase-upward profile $U(z, t)$ and phase-downward profile $D(z, t)$ :

$$
\begin{aligned}
U(z, t)= & \sum_{n, \sigma} \frac{1}{2}\left\{\left(A_{n \sigma}^{(1)}+B_{n \sigma}^{(2)}\right) \cdot \cos (\sigma \cdot t-n z)\right. \\
& \left.+\left(B_{n \sigma}^{(1)}-A_{n \sigma}^{(2)}\right) \cdot \sin (\sigma \cdot t-n z)\right\} \\
D(z, t)= & \sum_{n, \sigma} \frac{1}{2}\left\{\left(A_{n \sigma}^{(1)}-B_{n \sigma}^{(2)}\right) \cdot \cos (\sigma \cdot t+n z)\right. \\
& \left.+\left(B_{n \sigma}^{(1)}+A_{n \sigma}^{(2)}\right) \cdot \sin (\sigma \cdot t+n z)\right\} .
\end{aligned}
$$

Notice that the summations are made over selected frequency window and wave number window case by case. We shall
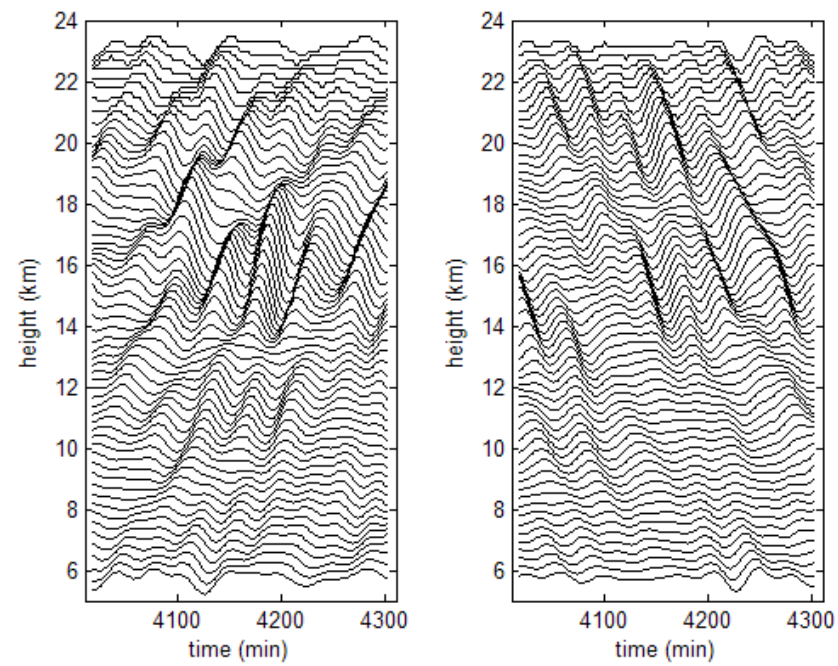

Fig. A1. Partial profile of winter zonal perturbation velocity obtained from Window- T2Z3: Data contributed by phase-upward waves was shown in the left panel, and data contributed by phasedownward waves was shown in the right panel.

analyze $U(z, t)$ and $D(z, t)$ instead of the original data set $\psi(z, t)$. Figure A1 demonstrates part of the phase upward profile (left panel) and phase downward profile (right panel) obtained from the zonal wind velocity profile in this study. Clearly, the left panel contains only phase-upward propagating wave, and the right panel contains only phase-downward propagating waves as expected.

\section{A2 Method of phase- and group-velocity tracing}

A Range-Time plot of the local power of fluctuation of $\Phi(z, t)$ (representing either $U(z, t)$ or $D(z, t))$ can be obtained by following procedure (Kuo et al., 1998): At each time step $t_{i}=i \Delta t$ and height $z_{k}=k \Delta z$, a time series of $N$ data points ( $N$ is chosen to be 15 in this paper) is defined by,

$$
\left\{\Phi_{\ell k}=\Phi\left(z_{k}, t_{\ell}\right), \ell=i-\frac{N}{2}, i-\frac{N}{2}+1, \ldots, i+\frac{N}{2}\right\}
$$

First, to remove the contribution from very long period waves, a linear trend of this time series (Eq. A5), $y=a t+b$, is obtained by the method of least square fitting, then a new time series (Eq. A6) is obtained from Eq. (A5) by subtracting its linear trend:

$$
\left\{\Phi_{\ell k}^{\prime}=\Phi_{\ell k}-a t_{\ell}-b, \ell=i-\frac{N}{2}, i-\frac{N}{2}+1, \ldots, i+\frac{N}{2}\right\}
$$

Then, the time series (A6) is Fourier analyzed to obtain the power $P_{k i}$ of the first harmonic. Considering $P_{k i}$ as an index of the strength of the wave activity at height $z_{k}$ and time $t_{i}$, we plot the matrix $\left[P_{k i}\right]$ as a Range-Time plot for investigation (we use contour plot to represent the Range-Time plot in this paper). Numerical simulation study (Kuo et al., 1998) showed that when a wave packet is propagating, packets with high regularity would exist in the Range-Time plot. 

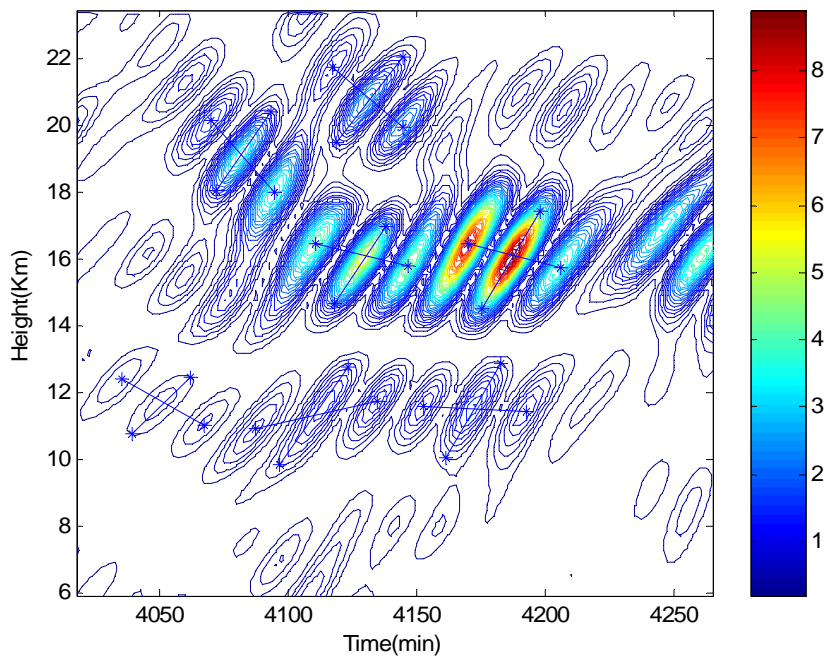

Fig. A2. The range time plot of $(\delta V)^{2}$ converted from left panel of Fig. A1 by the method of phase- and group-velocity tracing. Seven wave packets were identified and measured from this map.

It was shown that the same phase points of the characteristic wave component of the patch form a straight line called phase trajectory (phase line), while the centers of the neighboring patches form another straight line called energy trajectory (energy line). It was also noticed that the former determines the direction of the phase progression and the latter constitutes the direction of energy propagation. Therefore, the slope of the phase trajectory is just the phase velocity and the slope of the energy trajectory is the group velocity of the wave packet. Also, the horizontal distance between two consecutive packets (corresponding to the peaks and the dips in the oscillation profile) equals one half of the period of the characteristic wave component of the packet. Examples of the range time plots of $(\delta V)^{2}$ converted from left panel and right panel of Fig. A1 were shown in Figs. A2 and A3, respectively. We define a wave packet to consist of three consecutive patches so that its vertical phase velocity and group velocity can be unambiguously measured. We have identified and measured 7 and 6 wave packets from Figs. A2 and A3, respectively, and only one of them in Fig. A2 was type 2 wave packet.

\section{Appendix B}

On the relation between vertical flux of horizontal pseudo-momentum and momentum of atmospheric gravity waves

The vertical flux of the horizontal momentum $\left(\mathfrak{\Im}_{x z}, \mathfrak{\Im}_{y z}\right)$ is defined as the multiplication of the vertical group velocity $v_{g z}$ and the horizontal momentum density $\left(P_{x}, P_{y}\right)$, so

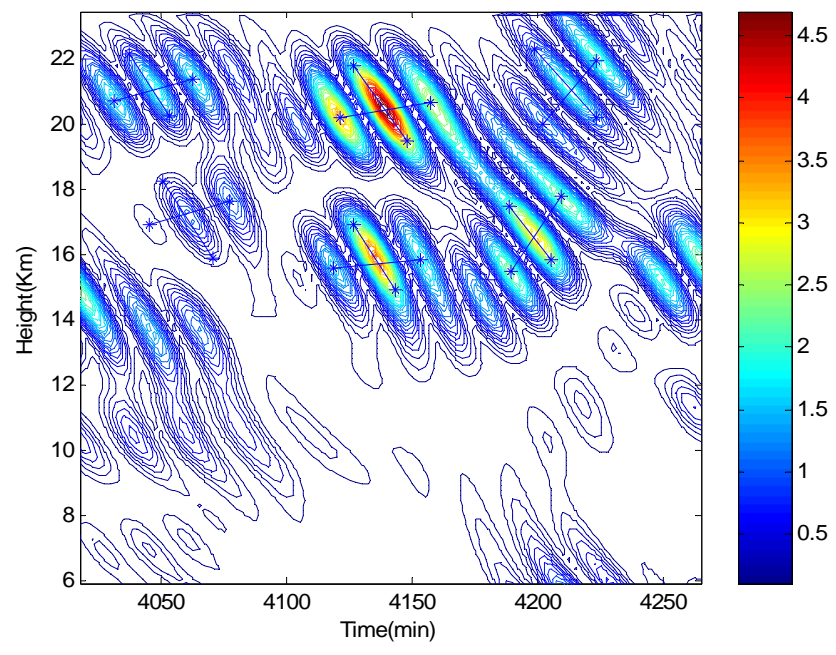

Fig. A3. The range time plot of $(\delta V)^{2}$ converted from right panel of Fig. A1 by the method of phase- and group-velocity tracing. Six wave packets were identified and measured from this map.

Eq. (7) (in the text) would yield Eq. (B1) for the expression of the horizontal pseudo-momentum density,

$\boldsymbol{P}_{h}=\left(P_{x}, P_{y}\right)=\rho_{0} \frac{E}{\omega}(k, \ell)$.

Similarly, Eq. (8) would yield Eq. (B2) for the expression of the horizontal momentum density,

$$
\tilde{\boldsymbol{P}}_{h}=\left(\tilde{P}_{x}, \tilde{P}_{y}\right)=\frac{\rho_{0}}{v_{g z}}\left(1-f^{2} / \omega^{2}\right)\left(\overline{u^{\prime} w^{\prime}}, \overline{v^{\prime} w^{\prime}}\right)
$$

In the linear theory of the atmospheric gravity waves, the polarization relation between the amplitudes of zonal component $U$ and the vertical component $W$ is known to be (Gossard and Hooke, 1975),

$$
\begin{aligned}
W & =\frac{i \omega}{N^{2}-\omega^{2}}\left(\frac{\omega^{2}-f^{2}}{\omega k+i f \cdot \ell}\right)\left(\frac{\partial}{\partial z}+\Gamma\right) U \\
& =\frac{\omega\left(\omega^{2}-f^{2}\right)}{N^{2}-\omega^{2}} \sqrt{\frac{m^{2}+\Gamma^{2}}{\omega^{2} k^{2}+f^{2} \ell^{2}}} \times e^{i\left(\theta_{1}+\theta_{2}\right)} \times U, \\
W & =\frac{i \omega}{N^{2}-\omega^{2}}\left(\frac{\omega^{2}-f^{2}}{\omega \ell-i f \cdot k}\right)\left(\frac{\partial}{\partial z}+\Gamma\right) V \\
& =\frac{\omega\left(\omega^{2}-f^{2}\right)}{N^{2}-\omega^{2}} \sqrt{\frac{m^{2}+\Gamma^{2}}{\omega^{2} \ell^{2}+f^{2} k^{2}}} \times e^{i\left(\theta_{3}+\theta_{2}\right)} \times V,
\end{aligned}
$$

or inversely,

$$
\begin{aligned}
& U=\frac{N^{2}-\omega^{2}}{\omega\left(\omega^{2}-f^{2}\right)} \sqrt{\frac{\omega^{2} k^{2}+f^{2} \ell^{2}}{m^{2}+\Gamma^{2}}} \times e^{-i\left(\theta_{1}+\theta_{2}\right)} \times W \\
& V=\frac{N^{2}-\omega^{2}}{\omega\left(\omega^{2}-f^{2}\right)} \sqrt{\frac{\omega^{2} \ell^{2}+f^{2} k^{2}}{m^{2}+\Gamma^{2}}} \times e^{-i\left(\theta_{3}+\theta_{2}\right)} \times W
\end{aligned}
$$




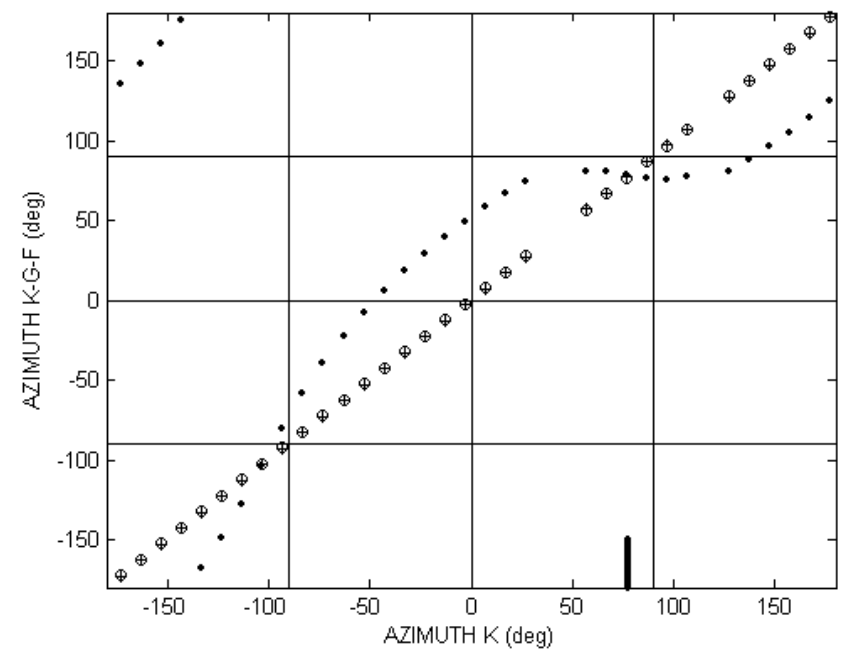

Fig. B1. The horizontal axis is the azimuth angles of the horizontal wave vector $(k, \ell)$, and the vertical axis are: cross denoted the azimuth angle of horizontal wave vector $(k, \ell)$; open circle denoted azimuth angle of the horizontal momentum vector $\left(\tilde{P}_{x}, \tilde{P}_{y}\right)$; dot denoted the azimuth angle of the horizontal group velocity $\left(v_{g x}, v_{g y}\right)$. The short thick vertical line indicated the azimuth angle of the winter mean wind velocity $(37.8,8.1) \mathrm{m} / \mathrm{s}$. The phase velocity and the wave period of this gravity wave were $30 \mathrm{~m} / \mathrm{s}$ and $143 \mathrm{~min}$, respectively.

where $\theta_{1}=\tan ^{-1}\left(\frac{\omega \cdot k}{f \cdot \ell}\right), \theta_{2}=\tan ^{-1}\left(\frac{m}{\Gamma}\right), \theta_{3}=\tan ^{-1}\left(\frac{\omega \cdot \ell}{-f \cdot k}\right)$ and $\Gamma \cong 3.2 \times 10^{-5} \mathrm{~m}^{-1} ; N \cong 1.05 \times 10^{-2} \mathrm{~s}^{-1}$ (BV frequency), $f \cong 8.31 \times 10^{-5} \mathrm{~s}^{-1}$ (inertial frequency); $\omega, k, \ell$ and $m$ are the intrinsic frequency, zonal-, meridional-, and vertical- wave number component respectively, and their relations with the observed wave frequency $\sigma$ are governed by the gravity wave dispersion Eqs. (1) and (2) in the text. The zonal-, meridional- and vertical components of fluctuation velocity $u^{\prime} v^{\prime}$ and $w^{\prime}$ of a wave mode with frequency $\sigma$, wave numbers $k, \ell$ and $m$ are given by,

$$
\begin{aligned}
u^{\prime}= & U \cos (k x+\ell y+m z-\sigma \cdot t) \\
= & \frac{N^{2}-\omega^{2}}{\omega\left(\omega^{2}-f^{2}\right)} \sqrt{\frac{\omega^{2} k^{2}+f^{2} \ell^{2}}{m^{2}+\Gamma^{2}}} \cdot W \\
& \cos \left(k x+\ell y+m z-\sigma \cdot t-\theta_{1}-\theta_{2}\right)
\end{aligned}
$$

$$
\begin{aligned}
v^{\prime}= & V \cos (k x+\ell y+m z-\sigma t) \\
= & \frac{N^{2}-\omega^{2}}{\omega\left(\omega^{2}-f^{2}\right)} \sqrt{\frac{\omega^{2} \ell^{2}+f^{2} k^{2}}{m^{2}+\Gamma^{2}}} \cdot W \\
& \cos \left(k x+\ell y+m z-\sigma \cdot t-\theta_{3}-\theta_{2}\right) \\
w^{\prime}= & W \cos (k x+\ell y+m z-\sigma \cdot t)
\end{aligned}
$$

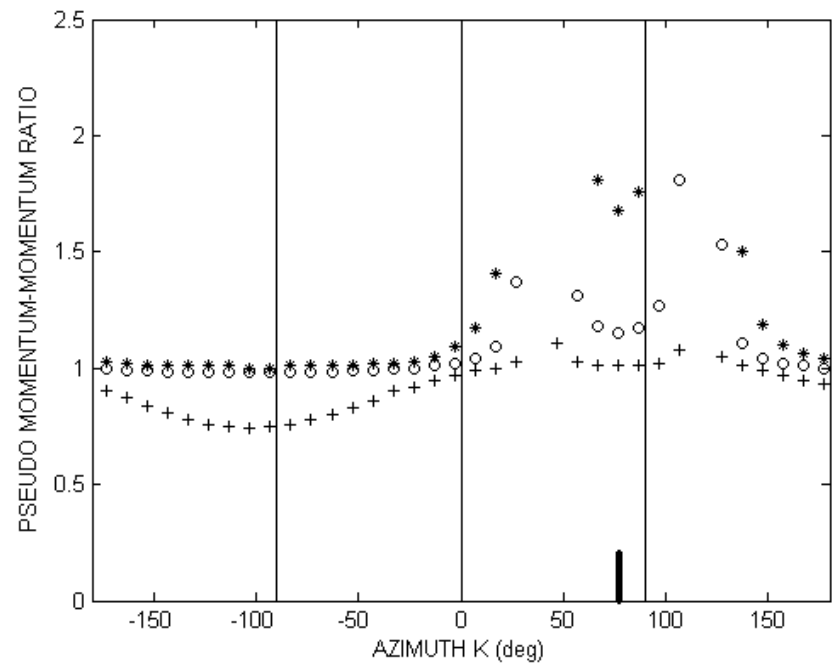

Fig. B2. The amplitude ratio between the horizontal pseudo momentum and the momentum (ratio= $=\left|\boldsymbol{P}_{h}\right| /\left|\tilde{\boldsymbol{P}}_{h}\right|$ ) as function of the azimuth angle of the corresponding AGW. Cross: $v_{p h}=30 \mathrm{~m} / \mathrm{s}$ and $\tau=45 \mathrm{~min}$; Open circle: $v_{p h}=30 \mathrm{~m} / \mathrm{s}$ and $\tau=143 \mathrm{~min}$; Star: $v_{p h}=30 \mathrm{~m} / \mathrm{s}$ and $\tau=300 \mathrm{~min}$.

Then, the time average of $u^{\prime} w^{\prime}$ can be expressed in terms of gravity wave parameters as,

$$
\begin{aligned}
\overline{u^{\prime} w^{\prime}}= & \frac{N^{2}-\omega^{2}}{\omega\left(\omega^{2}-f^{2}\right)} \sqrt{\frac{\omega^{2} k^{2}+f^{2} \ell^{2}}{m^{2}+\Gamma^{2}}} \cdot W^{2} \times \\
& \frac{. \cos \left(k x+\ell y+m z-\sigma \cdot t-\theta_{1}-\theta_{2}\right)}{\cos (k x+\ell y+m z-\sigma \cdot t)} \\
= & \frac{N^{2}-\omega^{2}}{\omega\left(\omega^{2}-f^{2}\right)} \sqrt{\frac{\omega^{2} k^{2}+f^{2} \ell^{2}}{m^{2}+\Gamma^{2}}} \times W^{2} \times \frac{1}{2} \cos \left(\theta_{1}+\theta_{2}\right),
\end{aligned}
$$

Similarly,

$$
\begin{aligned}
\overline{v^{\prime} w^{\prime}}= & \frac{N^{2}-\omega^{2}}{\omega\left(\omega^{2}-f^{2}\right)} \sqrt{\frac{\omega^{2} \ell^{2}+f^{2} k^{2}}{m^{2}+\Gamma^{2}}} \\
& \times W^{2} \times \frac{1}{2} \cos \left(\theta_{3}+\theta_{2}\right)
\end{aligned}
$$

The energy term in Eq. (A1) can be obtained from Eqs. (A4a) and (A4b) as follows,

$$
\begin{aligned}
E & =\overline{u^{\prime} u^{\prime}}+\overline{v^{\prime} v^{\prime}} \\
& =\left[\frac{N^{2}-\omega^{2}}{\omega\left(\omega^{2}-f^{2}\right)}\right]^{2} \times\left[\frac{\left(\omega^{2}+f^{2}\right)\left(k^{2}+\ell^{2}\right)}{m^{2}+\Gamma^{2}}\right] \times \frac{W^{2}}{2}
\end{aligned}
$$

The azimuth angle of the horizontal momentum obtained by Eqs. (B2), (B6) and (B7) is,

$$
\begin{aligned}
& \tilde{\phi}_{k h}=\tan ^{-1}\left(\frac{\tilde{P}_{x}}{\tilde{P}_{y}}\right)=\tan ^{-1}\left(\frac{\overline{u^{\prime} w^{\prime}}}{\overline{v^{\prime} w^{\prime}}}\right) \\
& =\sqrt{\frac{\omega^{2} k^{2}+f^{2} \ell^{2}}{\omega^{2} \ell^{2}+f^{2} k^{2}}} \cdot \frac{\cos \left(\theta_{1}+\theta_{2}\right)}{\cos \left(\theta_{3}+\theta_{2}\right)},
\end{aligned}
$$


and the azimuth angles of the horizontal wave vector $(k, \ell)$ and the horizontal group velocity $\boldsymbol{v}_{g h}\left(v_{g x}, v_{g y}\right)$ are given by Eqs. (B10) and (B11), respectively,

$\phi_{k h}=\tan ^{-1}\left(\frac{k}{\ell}\right)=\tan ^{-1}\left(\frac{P_{x}}{P_{y}}\right)$

$\phi_{g h}=\tan ^{-1}\left(\frac{v_{g x}}{v_{g y}}\right)$

We have made numerical calculations of Eqs. (B9), (B10) and (B11) under winter mean wind condition $(\bar{u}=37.8 \mathrm{~m} / \mathrm{s}$ and $\bar{v}=8.1 \mathrm{~m} / \mathrm{s}$ ) for monochromatic waves with period $\tau=45$, 143 and $300 \mathrm{~min}$, horizontal phase velocity $v_{p h}=20,30$ and $60 \mathrm{~m} / \mathrm{s}$, and azimuth angle $\phi_{k h}=7,17,27,-, 337,347$, 357 degrees. One example of the numerical result is shown in Fig. B1, which demonstrated that the values of the differences $\left|\tilde{\phi}_{k h}-\phi_{k h}\right|$ and $\left|\phi_{g h}-\phi_{k h}\right|$ were less than $3^{\circ}$ and $90^{\circ}$, respectively. Evidently, the direction of $\boldsymbol{v}_{g h}$ is not the same as that of $\boldsymbol{k}_{h}$ because of the existence of the strong winter wind. Another interesting factor is the amplitude ratio between the horizontal pseudo-momentum density $\boldsymbol{P}_{h}$ and the horizontal momentum density $\tilde{\boldsymbol{P}}_{h}$ (Eqs. B1 and B2),

Ratio $=\sqrt{\frac{\tilde{P}_{x}^{2}+\tilde{P}_{y}^{2}}{P_{x}^{2}+P_{y}^{2}}}$.

Example of the numerical result of Ratio vs. $\phi_{k h}$ for the waves with $v_{p h}=30 \mathrm{~m} / \mathrm{s}$ is shown in Fig. B2, which revealed that the ratio values were between 0.5 and 2.0. For the waves with $v_{p h}=60 \mathrm{~m} / \mathrm{s}$ the ratio values (not shown) were found to be closer to 1 than the case of $v_{p h}=30 \mathrm{~m} / \mathrm{s}$.

The above discussion was good for monochromatic waves. In the case of the superposition of multiple waves such as the case of a wave packet, the characteristic wave vector related to the vertical flux of horizontal momentum would be an ensemble average of the horizontal wave vectors of all the component waves.

Acknowledgements. This work is supported in part by the National Science Council of Taiwan under the contract number NSC 962111-M-238-001-MY3. The MU radar belongs to and is operated by the Research Institute for Sustainable Humanosphere (RISH) of Kyoto University.

Topical Editor C. Jacobi thanks two anonymous referees for their help in evaluating this paper.

\section{References}

Ebel, A., Manson, A. H., and Meek, C. E.: Short period fluctuations of the horizontal wind measured in the upper middle atmosphere and possible relationships to internal gravity waves, J. Atmos. Terr. Phys., 49, 385-401, 1987.

Eckermann, S. D. and Vincent, R. A.: Falling sphere observations of atmospheric gravity wave motions in the upper stratosphere over Australia, Pure Appl. Geophys., 130, 509-532, 1989.
Fritts, D. C. and Alexander, M. J.: Gravity wave dynamics and effects in the middle atmosphere, Rev. Geophys., 41(1), 1003, doi:10.1029/2001RG000106, 2003.

Gavrilov, N. M., Fukao, S., Nakamura, T., and Tsuda, T.: Statistical analysis of gravity waves observed with the middle and upper atmosphere radar in the middle atmosphere 2, Waves propagated in different directions, J. Geophys. Res., 102(D12), 13433-13440, 1997.

Gossard, E. E. and Hooke, W. H.: Waves in the Atmosphere, Elsevier, New York, p. 98, 1975.

Hirota, I. and Niki, T.: Inertia-gravity waves in the troposphere and stratosphere observed by the MU radar, J. Meteorol. Soc., Japan, 64, 995-999, 1986.

Kuo, F. S., Lue, H. Y., Huang, C. M., Lo, C. L., Liu, C. H., Fukao, S., and Muraoka, Y.: A study of velocity fluctuation spectra in the troposphere and lower stratosphere using MU radar, J. Atmos. Terr. Phys., 54(1), 31-48, 1992.

Kuo, F. S., Lue, H. Y., and Luo, C. L.: Physical parameters of gravity wave packet propagation contained in radar RTI plots., J. Atmos. Terr. Phys., 60(4), 455-460, 1998.

Kuo, F. S., Röttger, J., and Lue, H. Y.: Propagation of gravity wave packets in the lower atmosphere observed by the SOUSYSvalbard radar, Chinese J. Phys., 41(4), 309-325, 2003.

Kuo, F. S. and Röttger, J.: Horizontal wavelength of gravity wave in the lower atmosphere measured by the SOUSY Svalbard Radar, Chinese J. Phys., 43(3-1), 464-480, 2005.

Kuo, F. S., Lue, H. Y., and Fern, C. L.: Phase and group velocity tracing analysis of projected wave packet motion along oblique radar beams - qualitative analysis of QP echoes, Ann. Geophys., 25, 77-86, 2007, http://www.ann-geophys.net/25/77/2007/.

Kuo, F. S., Lue, H. Y., Fern, C. L., Röttger, J., Fukao, S., and Yamamoto, M.: Studies of vertical fluxes of horizontal momentum in the lower atmosphere using the MU-radar, Ann. Geophys., 26, 3765-3781, 2008, http://www.ann-geophys.net/26/3765/2008/.

Manson, A. H. and Meek, C. E.: Gravity wave propagation characteristics $(60-120 \mathrm{~km})$ as determined by the Saskatoon MF Radar (GRAVNET) system: $1983-85$ at $52^{\circ} \mathrm{N}, 107^{\circ} \mathrm{W}$, J. Atmos. Sci., 45, 932-946, 1988.

Meek, C. E., Reid, I. M., and Manson, A. H.: Observations of mesospheric wind velocities, 1 . Gravity wave horizontal scales and phase velocities determined from spaced wind observations, Radio Sci., 20, 1363-1382, 1985.

Nakamura, T., Tsuda, T., Yamamoto, M., Fukao, S., and Kato, S.: Characteristics of gravity waves in the mesosphere observed with the middle and upper atmosphere radar, 2. Propagation Direction, J. Geophys. Res., 98(D5), 8911-8923, 1993.

Sato, K.: A statistical study of the structure, saturation and sources of inertia-gravity waves in the lower stratosphere observed with the MU radar, J. Atmos. Terr. Phys., 56, 755-774, 1994.

Thomas, L., Worthington, R. M., and McDonald, A. J.: Inertiagravity waves in the troposphere and lower stratosphere associated with a jet stream exit region, Ann. Geophys., 17, 115-121, 1999, http://www.ann-geophys.net/17/115/1999/.

Tsuda, T., Sato, S., Yokoi, T., Inoue, T., Yamamoto, M., VanZandt, T. E., Fukao, S., and Sato, T.: Gravity waves in the mesosphere observed with the MU radar, Radio Sci., 26, 1005-1018, 1990.

Vincent, R. A. and Fritts, D. C.: A climatology of gravity wave motions in the mesosphere region of Adelaide, Australia, J. Atmos. Sci., 44, 748-760, 1987. 
Vincent, R. A. and Reid, I. M.: HF Doppler measurements of mesospheric gravity wave momentum fluxes, J. Atmos. Sci., 40(5), 1321-1333, 1983.

Yamamoto, M., Tsuda, T., and Kato, S.: Gravity waves observed by the Kyoto meteor radar in 1983-1985, J. Atmos. Terr. Phys., 48, 597-603, 1986.
Yamanaka, M. D., Fukao, S., Matsumoto, H., Sato, T., Tsuda, T., and Kato, S.: Internal gravity wave selection in the upper troposphere and lower stratosphere observed by the MU radar: preliminary results, Pure Appl. Geophys., 130, 481-495, 1989. 